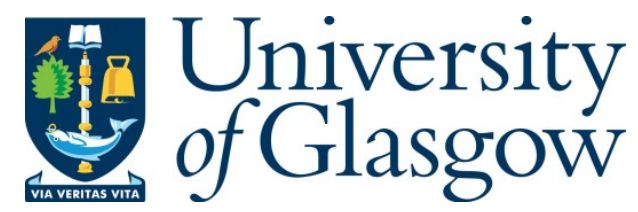

Kowalski, A. F., Cauzzi, G., and Fletcher, L. (2015) Optical spectral observations of a flickering white-light kernel in a C1 solar flare. Astrophysical Journal, 798(2), 107.

Copyright (C) 2015 American Astronomical Society

Version: Accepted

http://eprints.gla.ac.uk/107612

Deposited on: 03 July 2015

Enlighten - Research publications by members of the University of Glasgow http://eprints.gla.ac.uk 


\title{
Optical Spectral Observations of a Flickering White-Light Kernel in a C1 Solar Flare
}

\author{
Adam F. Kowalski \\ NASA Postdoctoral Program Fellow, Goddard Space Flight Center, Code 671, 8800 \\ Greenbelt Rd., Greenbelt, MD 20771 USA \\ adam.f.kowalski@nasa.gov \\ and \\ Gianna Cauzzi \\ INAF-Osservatorio Astrofisico di Arcetri, Firenze, Italy \& National Solar Observatory, \\ Sunspot NM, USA \\ and \\ Lyndsay Fletcher \\ SUPA School of Physics and Astronomy, University of Glasgow, Glasgow G12 8QQ, UK
}

Received — 


\begin{abstract}
We analyze optical spectra of a two-ribbon, long duration C1.1 flare that occurred on 18 Aug 2011 within AR 11271 (SOL2011-08-18T15:15). The impulsive phase of the flare was observed with a comprehensive set of space-borne and ground-based instruments, which provide a range of unique diagnostics of the lower flaring atmosphere. Here we report the detection of enhanced continuum emission, observed in low-resolution spectra from $3600 \AA$ to $4550 \AA$ acquired with the Horizontal Spectrograph at the Dunn Solar Telescope. A small, $\leq 0^{\prime \prime} .5\left(10^{15}\right.$ $\mathrm{cm}^{2}$ ) penumbral/umbral kernel brightens repeatedly in the optical continuum and chromospheric emission lines, similar to the temporal characteristics of the hard X-ray variation as detected by the Gamma-ray Burst Monitor (GBM) on the Fermi spacecraft. Radiative-hydrodynamic flare models that employ a nonthermal electron beam energy flux high enough to produce the optical contrast in our flare spectra would predict a large Balmer jump in emission, indicative of hydrogen recombination radiation from the upper flare chromosphere. However, we find no evidence of such a Balmer jump in the bluemost spectral region of the continuum excess. Just redward of the expected Balmer jump, we find evidence of a "blue continuum bump" in the excess emission which may be indicative of the merging of the higher order Balmer lines. The large number of observational constraints provides a springboard for modeling the blue/optical emission for this particular flare with radiative-hydrodynamic codes, which are necessary to understand the opacity effects for the continuum and emission line radiation at these wavelengths.
\end{abstract}




\section{Introduction}

Blue/optical flare spectra contain a wealth of information on the response of the lower stellar atmosphere to flare energy input, which can be diagnosed through line and continuum measurements. However, spectroscopic observations of the white-light (WL) optical continuum in solar flare kernels are few and far between, dating back to a handful of spectra obtained over 30 years ago, primarily with the Universal Spectrograph at the Sacramento Peak Observatory (Machado \& Rust 1974; Neidig 1983; Donati-Falchi et al. 1984, see also Hiei (1982)). The few spectra that exist show diverse continuum properties. Neidig (1983) compiled three of these spectra, looking at the Balmer jump region $(\lambda=3646$ $\AA$, corresponding to the long-wavelength edge of the recombination continuum onto hydrogen $n=2$ ). The Balmer jump appeared in one event, not at all in the second and, apparently, $50 \AA$ redward of the Balmer edge wavelength in the third. These spectra have been modeled as a combination of two continuum emission components: a free-bound hydrogen Balmer recombination spectrum and a $\mathrm{H}^{-}$recombination spectrum (Hiei 1982 , Neidig 1983; Donati-Falchi et al. 1985; Boyer et al. 1985; Neidig et al. 1994). It has also been suggested that optical emission attributed to $\mathrm{H}^{-}$is entirely due to hydrogen Paschen recombination radiation (Neidig \& Wiborg 1984), but Boyer et al. (1985) were unable to find a satisfactory answer from a Paschen (or Balmer) recombination spectrum fit to their flares, and instead argued for $\mathrm{H}^{-}$emission. A claimed third continuum component at $\lambda<4000 \AA$ Zirin 1980; Zirin \& Neidig 1981), has been shown to result from the merging of Stark-broadened hydrogen line wings, creating pseudo-continuum emission between the Balmer lines, and between the expected Balmer edge wavelength and the bluest observed Balmer line (Donati-Falchi et al. 1985).

Ideas about the lower flare atmosphere obtained from these spectra have been reached primarily through comparison with static, isothermal, constant density models. A hydrogen 
recombination model has been used to infer temperatures of $7000-10,000 \mathrm{~K}$ and electron densities of $\sim 1-5 \times 10^{13} \mathrm{~cm}^{-3}$, which gives an origin in the lower chromosphere $(\sim 1000$ $\mathrm{km}$ above the quiet-Sun $\tau_{5000}=1$ level). Increased emission from $\mathrm{H}^{-}$during flares on the other hand implies a temperature increase of the upper photosphere $(\sim 50-300 \mathrm{~km}$ above the quiet-Sun $\tau_{5000}=1$ level) by at least several hundred K (see Neidig 1989, for a review). However, the limitation of information derived from these models is that the important line and all continuum components are assumed to originate from a common uniform, static, one-dimensional atmospheric layer, a rather crude approximation long discredited by observations (e.g. Cauzzi et al. 1996; Falchi \& Mauas 2002). With this type of analysis, it is not possible to constrain a combination of emission mechanisms from dynamic gradients in the temperature, density, pressure, and ionization.

Among the various heating mechanisms which have been considered to produce the white-light emission, the most commonly-cited is the bombardment of the chromosphere by nonthermal deka-keV electrons (Hudson 1972; Abbett \& Hawley 1999), leading either to direct heating of the photosphere or production of free-bound emission in the mid chromosphere (which can also drive UV radiative backwarming, e.g., Machado et al. 1989, Hawley \& Fisher 1992). Electrons are favoured because of the close relationship between the timing and spatial locations (i.e., kernels) of white-light and hard X-ray emission (Hudson et al. 1992). Energetically, this tends to require all electrons down to around $20 \mathrm{keV}$ to excite the radiation, but it is not clear that these electrons can reach the altitudes required to produce the continuum: certainly not the photosphere, and even reaching the mid chromosphere can be challenging (Fletcher et al. 2007). Other possible heating mechanisms include bombardment by non-thermal MeV protons (Švestka 1970; Machado et al. 1978), heating by Alfvén waves (Fletcher \& Hudson 2008, Russell \& Fletcher 2013), or a heated compression wave propagating towards the photosphere (Livshits et al. 1981). However, until the optical spectrum of flares has been properly and systematically characterized, and 
compared with model predictions (e.g. the radiation hydrodynamics models of Allred et al. 2005) it will not be possible to precisely identify the heating mechanism(s) responsible.

White-light emission was once thought to only originate in large flares, but now has been observed from $\sim \mathrm{C} 2$ through X-class (Hudson et al. 2006; Fletcher et al. 2007; Jess et al. 2008; Kretzschmar 2011). Unfortunately, the focus on high spatial and temporal resolution in modern solar observations means that almost all current white-light data are solely from narrow-band (e.g., G-band) or broad-band (TRACE/WL) images. There is very little broad-band spectroscopy (color), or information about optical emission line behavior. If the white-light spectrum is known, energetics can be constrained (Neidig et al. 1994; Kerr \& Fletcher 2014), and a direct comparison made with the nonthermal particle power deduced from hard X-ray observations (Metcalf et al. 2003; Fletcher et al. 2007) and with the spectral models from each of the proposed heating mechanisms. In recent times, this has only been done for the Sun using available 3-color (red/green/blue) filter measurements, all at wavelengths longer than the Balmer edge. For example, a recent superposed epoch analysis by Kretzschmar (2011) of Sun-as-a-Star three-color measurements made at the peaks of flares from upper-C to X-class shows that the data are consistent with a $T \sim 9000$ K blackbody continuum. Using the Hinode Solar Optical Telescope, Kerr \& Fletcher (2014) and Watanabe et al. (2013) found consistency with a much lower temperature blackbody (in the former, free-bound continuum was also possible, but much more demanding energetically). It is interesting that a hot (9000 K) blackbody continuum component has never been reproduced in radiative-hydrodynamic models that employ a realistic heating model, although it is well-known to dominate the optical spectra and broadband energetics during flares on active M dwarf stars (Hawley \& Pettersen 1991; Fuhrmeister et al. 2008; Kowalski et al. 2013).

In this paper, we present the first spatially and temporally resolved spectra with 
broad-wavelength $(\sim 3600-4550 \AA)$ and moderate spectral resolution $(\mathrm{R} \sim 4,000)$ coverage of a white-light kernel, obtained during a small C-class flare. Simultaneous imaging spectroscopy in photospheric and chromospheric lines allows a clear framing of the white-light emission with respect to the global spatial and temporal development of the flare, which was not possible at the time of the earlier spectroscopic investigations of white-light flares. Indeed, as remarked in Neidig (1989), older broadband spectra were never obtained on the brightest flaring kernel during the impulsive phase. Further, a modern day investigation of the continuum emission is especially important because of the availability of complementary data in the UV and EUV with the Solar Dynamics Observatory's Atmospheric Imaging Assembly (Lemen et al.|2012), as well as nonthermal hard X-rays from the Fermi Gamma-ray Burst Monitor (GBM; Meegan et al. 2009). These data will allow the nonthermal electron energy and number flux to be constrained, to be used as input to future flare models, allowing the heating and excitation mechanisms to be tested.

Section 2 describes the observations and spectral data reduction, and Section 3 describes the white-light detection. In Section 4, we present the chromospheric emission line properties. In Section 5, we summarize our observations and discuss some of their physical implications, and how they compare with isothermal slab and hydrodynamic flare models. Section 6 contains several conclusions from the data.

\section{Observations and Data Reduction}

The active region NOAA 11271 (N16.5E42.1) produced a C1.1 flare on August 18, 2011 with a GOES 1-8 $\AA$ peak at approximately UT 15:15 (SOL2011-08-18T15:15). The flare exhibited one extended ribbon in weak-field plage region, and much more compact, short ribbons or groups of footpoints in the sunspot umbra/penumbra (Figure 1). It had a 
fairly long decay in GOES, with several hard X-ray peaks, but we concentrate here on the largest impulsive burst at around UT 15:09:30. Unfortunately, RHESSI was in the South

Atlantic Anomaly during the main burst and the optical observations, but Fermi registered the event from around $6-25 \mathrm{keV}$, allowing for a comparison of the optical data with the $\mathrm{X}$-ray impulsive phase.

We observed this flare with a comprehensive set of instruments at the Dunn Solar Telescope (DST) of the National Solar Observatory, employing adaptive optics (Rimmele 2004). Region NOAA 11271 was monitored continuously between UT 14:10 and 16:20, with some brief interruptions to re-point the instruments. Atmospheric conditions were clear, and seeing conditions remained good and fairly stable throughout. The blue light from the DST was directed to the Horizontal Spectrograph (HSG) with a setup described in Section 2.3, whereas the red light was directed to the Interferometric Bidimensional Spectrometer (IBIS, Cavallini 2006). Preliminary results have been presented in Fletcher et al. (2013), and a comprehensive multi-wavelength analysis will follow in a subsequent paper. In this paper, we focus on the WL detection and the optical emission line characteristics compared to the X-ray impulsive phase.

\subsection{X-Ray Data}

We obtained the Fermi/GBM and GOES 1-8 $\AA$ (1.5-12.4 keV) light curves using the IDL SolarSoft OSPEX software. The Fermi/GBM CSPEC file from the NaI detector \#5 (the most sunward facing) was used to produce a $14.58-20.70 \mathrm{keV}$ hard X-ray count flux light curve. This light curve was detrended to remove the long-term background modulation, and a small residual pre-flare enhancement was also subtracted. The live time was $4.07 \mathrm{~s}$ until 15:08:56, after which an automatic trigger initiated with a live time of $1.02 \mathrm{~s}$ until about 15:19. We bin all data to $4.07 \mathrm{~s}$ and the count flux is normalized to the 
peak value of 0.33 counts $\mathrm{cm}^{-2} \mathrm{~s}^{-1} \mathrm{keV}^{-1}$ at 15:09:25. The hard X-ray light curve is shown in Figure 2 and its properties are described in Sections 3 and 4.

\subsection{IBIS data}

IBIS imaged a field of view (FOV) of $98^{\prime \prime}$ diameter with a $0^{\prime \prime} .098$ pixel size, sampling the line profiles of Fe I $5434 \AA$ (26 steps), H $\alpha$ (30 steps), and Ca II $8542 \AA$ (29 steps). The cadence for the full spectral sequence was $\sim 17 \mathrm{~s}$. We use here mostly the images acquired in the red wing of $\mathrm{H} \alpha$ to examine the flare kernel development. Figure 1 gives an overview of the region and the flare as observed with IBIS. The two small spots seen in the broadband image as sharing a penumbra were of the same polarity as the leading spot (not in the field of view), and coalesced and grew over the course of two days in the central portion of the active region. This created a compact magnetic neutral line against more sparse plage elements of the following polarity, barely noticeable in the bottom part of the broadband image as bright small features. The co-temporal $\mathrm{H} \alpha+1.2 \AA$ image (top right) shows these plage elements much more clearly than in the broadband, due to the relative lack of contrast of convective features at this wavelength, combined with the enhanced temperature of magnetic elements in the mid photosphere (Leenaarts et al. 2006).

The two bottom images clearly show the flare ribbons. Flare emission in the far red wing of $\mathrm{H} \alpha$ usually displays a very impulsive character and strong spatial and temporal correlation with hard X-ray bursts (Kurokawa et al. 1988; Cauzzi et al. 1995; Radziszewski et al. 2011; Deng et al. 2013). Such characteristics are attributed to both local heating and, especially, to the downward moving front of the chromospheric condensation, driven in turn by intense, localized heating such as would be caused by electron precipitation (e.g. Ichimoto \& Kurokawa 1984; Canfield et al. 1990). For this reason, the position of the flare ribbons (or kernels) as imaged in such wavelengths has often been used to identify 
the electron precipitation site. The bottom panels of Figure 1 clearly display the motion of the plage flare ribbon, which proceeds further into the weak field region as the flare

progresses, tracing the successive involvement of magnetic field during the flare (e.g. Falchi et al. 1997). On the contrary, the spot ribbon does not display any lateral displacement, but rather a succession of bright kernels along a very defined direction (with some repeated episodes in the same kernels). This is most likely due to a very strongly convergent field joining the weak plage to the spot, and determines the extremely narrow ribbon width, which in various positions approaches the diffraction limit of our observations, $\sim 200 \mathrm{~km}$. Such a property has been observed in other events (always involving a spot ribbon), and can provide important constraints to the standard thick target beam interpretation of solar flares (Krucker et al. 2011; Sharykin \& Kosovichev 2014).

\subsection{Blue/Optical Spectroscopic Data}

The goal of the optical spectroscopy was to obtain spectra with maximum wavelength coverage while including the Balmer edge wavelength at $\lambda=3646 \AA$. To achieve this, we employed a customized setup of the HSG on the DST. The solar spectrum from 3500-4560 $\AA$ A was imaged over four CCD's in order from bluest to reddest, respectively: ccc06, ccc01, ccc07, ccc08 with instrumental parameters given in Table 1. However, only a portion of the spectral range within each CCD was useable, as will be described in Section 2.4. A rastering slit with dimensions of $170^{\prime \prime} \times 0^{\prime \prime} .67$ was employed with 20 slit positions. The leftmost slit position in the field of view of Figure 1 is referred to as the first slit position throughout the paper. The step-size between each slit position was $0^{\prime \prime} .6775$, giving a total raster extent of $170^{\prime \prime} \times 13^{\prime \prime} .5$, and the total cycle time was $\sim 21 \mathrm{~s}$. The spectra were obtained with the slit oriented perpendicular to the horizon (at the parallactic angle), to ensure that image displacements due to differential refraction would align with the spectrograph slit. Such 
displacements are clearly noticed in high spatial resolution solar observations (Reardon 2006), and are of the greatest relevance at the blue wavelengths we employ in this study. The raster direction was perpendicular to the slit; this made for an orientation of the field of view in Figure 1, Figure 4, and Figure 5 different from the standard orientation with solar North up.

We also obtained high spatial sampling images $\left(\sim 0^{\prime \prime} .075\right.$ pixel $\left.^{-1}\right)$ with the slit jaw camera through the NBF4170 filter, typically employed with the ROSA instrument (Jess et al. 2010). This filter is centered on $\lambda=4170 \AA$ with a bandpass of $52 \AA$ and an exposure time of $10 \mathrm{~ms}$. The slit jaw images allowed us to accurately determine the position of the slit on the Sun.

\subsection{Spectroscopic Data Reduction}

The spectroscopic data reduction was performed using standard IRAF ${ }^{1}$ and IDL routines. We corrected all images for dark current. De-focused quiescent solar spectra obtained away from the active region were used for flat field, wavelength, and intensity calibration, which is described in detail in Appendix A. The reference spectrum for calibration was the disk-center absolute solar intensity spectrum obtained with the Fourier Transform Spectrometer (FTS) with spectral resolution $\mathrm{R}=350000($ Neckel 1999). The nominal dispersions for each camera are given in Table 1, but we note that, as a compromise between spatial scale along the slit and exposure times, the slit width was fixed at $90 \mu \mathrm{m}$, corresponding to an actual spectral resolution of $0.9-1.2 \AA$ at $4300 \AA \quad(\mathrm{R} \sim 4000)$. We

\footnotetext{
${ }^{1}$ IRAF is distributed by the National Optical Astronomy Observatory, which is operated by the Association of Universities for Research in Astronomy (AURA) under cooperative agreement with the National Science Foundation.
} 
converted the $2 \mathrm{D}$ spectra to intensity $\left(I_{\lambda, \mu=0.74} ; \operatorname{ergs} \mathrm{cm}^{-2} \mathrm{~s}^{-1} \mathrm{sr}^{-1} \AA^{-1}\right)$ by accounting for limb darkening, instrumental sensitivity, and the atmospheric extinction. The spectra from each CCD were aligned and interpolated to a common pixel scale $\left(0^{\prime \prime} .39\right.$ pixel $\left.^{-1}\right)$. Wavelength-dependent shifts of $0.5-2$ pixels were applied to account for differential refraction within each camera's spectral range.

As demonstrated in Figure 3, we found a satisfactory agreement between the observed quiescent spectrum (obtained at approximately $(x, y)=\left(38^{\prime \prime}, 20^{\prime \prime}\right)$ in Figure 1; i.e., between the plage regions) and the disk-center reference FTS spectrum binned to the HSG spectral resolution and converted to $I_{\lambda, \mu=0.74}$. Unfortunately, the current DST optical path is not optimized for work over very broad wavelength ranges, and suffers from chromatic aberration. The bluemost camera suffered the most from this problem, displaying differential spatial and spectral focus in large portions of its range, except for pristine focus in the interval $\lambda=3654-3674 \AA$. Interpretation of the data outside this spectral region is especially problematic in the umbral regions where there are sharp spatial gradients in intensity. At $\lambda<3600 \AA$ and $\lambda>3740 \AA$, the chromatism becomes severe and spectral and spatial features are largely defocused. In the figures, we show the spectral range from $\lambda=3600-3740 \AA$; although characterization is not robust through this entire spectral range, the degree to which we can detect the continuum and line features is satisfactory. Besides these problems, the chromatism was evident in the ccc07 camera from 4016-4200 $\AA$, such that solar features at $\lambda=4200 \AA$ were sharper than the features at $\lambda=4016 \AA$. The focus differs slightly among the four CCD's, with excellent overall focus in ccc08, and poor overall focus in ccc01.

Based on comparisons of our data to the disk-center FTS spectrum, we found the useable wavelength ranges are the following: $3654-3674 \AA$ for ccc06, $3830-3978 \AA$ for ccc01, 4085-4125 $\AA$ for ccc07, and $4213-4553 \AA$ for ccc08. 


\section{White-Light Detection}

Historically, many different parameters have been used to characterize white-light emission in solar flares. Jess et al. (2008) has demonstrated that ambiguity can result if the quantities are not precisely defined. Here, we calculate the enhancement, excess intensity, and contrast, in order to allow a meaningful comparison to the variety of measurements in older literature.

\subsection{Continuum enhancement}

The enhancement (or ratio) images are obtained by dividing the intensity at the time of the flare by the pre-flare intensity at UT 15:07:24 at the same spatial location. Figure 4 shows enhancement images for the continuum $(\lambda \sim 4170 \AA$; top panels $)$ and at $\mathrm{H} \delta$ line-center $(\lambda=4101 \AA$; bottom panels). In Figure 5 we also show a similar field of view extracted from the IBIS $\mathrm{H} \alpha+1.2 \AA$ data at multiple times, which allows the red wing kernels to be compared to the location of the blue/optical enhancements.

The enhancement images in the continuum are quite noisy, with many scattered small scale features whose intensity changes by a few percent between time steps. This is due mostly to transparency and seeing fluctuations (which have the largest effect in areas of large intensity gradients and cannot be removed from slit spectra), as well as to general evolution of the structures, for example the slow variation of brightness in the plage elements at the bottom of the panels. However, the very bright small feature at the leftmost slit position, indicated by the white arrow in the 15:09:30 panel, is a genuine candidate for a WL enhancement in the umbral region. Indeed, this feature appears at the same position along the slit as the umbral flare kernel shown in the 15:09:34 panel of Figure 1 and, most importantly, its temporal evolution follows closely that of the umbral 
flare kernels as observed both in the core of $\mathrm{H} \delta$ (bottom panels of Figure 4) and in the wing of $\mathrm{H} \alpha$ (Figure 5). In particular, the WL brightening is readily discernible at the same spatial location in three consecutive images, 15:09:08, 15:09:30 and 15:09:51, reaching an enhancement $\geq 1.25$ at 15:09:51 UT. After fading away, it re-appears briefly about 1 min later, at 15:10:54, again with an enhancement of $\sim 1.25$, consistent with the repeated appearance of the umbral kernel in the $\mathrm{H} \alpha$ wing images at 15:11 (Figure 5). Figure 2 shows the Fermi hard X-ray $(15-21 \mathrm{keV})$ and GOES soft X-ray $(1-8 \AA)$ light curves with the times of the simultaneous umbral kernel enhancements observed in the blue/optical continuum and at $\mathrm{H} \alpha+1.2 \AA$ as grey vertical bars. This shows that the kernel "flickering" occurs in the impulsive phase well before the maximum soft X-ray emission and is generally consistent with the well-established temporal coincidence of hard X-ray and the white-light emission (Kane et al. 1985; Hudson et al. 1992; Neidig \& Kane 1993; Fletcher et al. 2007).

As commented on in Section 2.2, the spatial extent of the flare umbral kernels is very small. In particular, the kernel intersected by the slit at 15:09:08 is roughly circular in shape, with a diameter of 5 IBIS pixels, i.e. $\leq 0^{\prime \prime} .5$. This is consistent with the size of the WL enhancement shown in Figure 4, which extends to at most two pixels along the slit, while being detectable at a significant level only in the first slit position of the raster. Thus, the WL kernel appears spatially unresolved in the HSG data. Using the circular figure from the IBIS data, we find an upper limit for the area encompassing the $\mathrm{H} \alpha$ wing excess intensity of $\sim 10^{15} \mathrm{~cm}^{2}\left(1^{\prime \prime}=734 \mathrm{~km}\right)$. This is comparable to the areal coverage of the WL kernel observed by Jess et al. (2008) during a C2.0 flare, at $\lambda=3953.7 \pm 5 \AA$. In Section 5 . we show that the resolved area is important for estimating the actual intensity values. 


\subsection{Excess $\left\langle I_{\lambda}\right\rangle$}

The excess spatially averaged intensity, or excess $\left\langle I_{\lambda}\right\rangle$, is defined as the pre-flare intensity at 15:07:24 subtracted from the flare intensity and averaged over the spatial extent of 3 pixels $\left(1^{\prime \prime} .2 \times 0^{\prime \prime} .67\right)$ centered on the brightest pixel. The averaging was done to account for slight residual spatial misalignments of the four CCDs and to account for the PSF of the slit. We note that, although the excess intensity can be used directly for calculating the flare energetics (see Section 5), it does not directly relate to an intensity from a given emission mechanism unless the emission is completely optically thin'? It is however useful for detecting a low level of flare emission.

In Figure 6, we show the time-evolution of the excess emission in continuum regions within each camera at the umbral kernel position. Note that these are lower limits to the excess intensity since the umbral kernel is unresolved in the spectra (but see Section 5). The two episodes of statistically significant continuum brightenings at 15:09:30-15:09:51 and at 15:10:54 are highlighted with grey bars in Figure 6, the continuum excess is evident across the full spectral range. Histograms of the excess intensity value per pixel at 15:09:30 at selected continuum wavelength regions and in $\mathrm{H} \gamma$ are shown in Figure 7, demonstrating that the intensity in the umbral kernel is well outside the spatial fluctuations in the data (e.g., top panel of Figure 4). The intensity values in the three pixels that are averaged for the umbral kernel are indicated by vertical dotted lines; at least one of each set of three pixels is $\geq 3 \sigma$ of the distribution.

As mentioned, atmospheric seeing variations can induce fluctuations in intensity over time, with particularly strong effects near large gradients in intensity, such as within the

\footnotetext{
${ }^{2}$ As noted by Acampa et al. (1982), the excess emission is a metrological quantity; see also the discussion in Kerr \& Fletcher (2014).
} 
umbra. In the bottom panel of Figure 6, we show the average excess intensity variations from a non-flaring umbral region with an average intensity level and gradient similar to that of the umbral kernel. The standard deviation of the light curve of the non-flaring umbral excess gives an estimate of the statistical fluctuation; we adopt this variation for the error bars in the top panel of Figure 6. The excess values within the time ranges indicated by the grey bars in the top panel of Figure 6 occur at a confidence level of $4.5-5 \sigma$ in the four continuum regions. The continuum variations in this location during times outside the grey bars are not significant enough to conclude they are true enhancements.

Any similar continuum excess outside the umbral region (at other slit positions) would give a lower enhancement, and may not be detectable from visual inspection of Figure 4 . Therefore, we performed a systematic search over all times and all slit positions, requiring that the excess $\mathrm{H} \gamma$ and the excess continuum intensity at $\lambda=3654-3674 \AA$ exceed a significance of $5 \sigma$ and $3 \sigma$, respectively, where $\sigma$ is determined from the spatial variation of the excess along the slit at each slit position (as in Figure 7). In addition to the umbral kernel, we find a candidate continuum increase at 15:09:47 in the 19th slit position in the plage ribbon, coincidentally within the time range of the umbral enhancement. However, this signal is only significant at a $3 \sigma$ level in the bluemost camera and $2 \sigma$ in the other cameras. At this level, we cannot conclude that this is a bona-fide WL excess.

Figure 8 shows the full spectral range of the spatially averaged excess intensity at 15:09:30 in the first raster slit position for the umbral kernel. The umbral kernel spectra at 15:09:51 and 15:10:54 exhibit similar continuum properties and are not shown. The rising intensity from $\lambda=4000 \AA$ to $\lambda=4200 \AA$ is probably a residual effect of the strong intensity gradient experienced by this camera from blue to red wavelengths (due to both the solar spectrum and the detector spectral response), made evident because of problems with non-linearity at the low counts of the sunspot spectrum. Also, the strong chromatic 
aberration across this chip (see Section 2.4) might cause the mixing of adjacent spectra from vastly different features, a problem of further relevance in the case of strong spatial intensity gradients as in the spot-penumbra transition. This likely results in the apparent continuum jump at $\lambda=4200 \AA$ in Figure 8 (between the two red most cameras), preventing a detailed slope characterization, and we display it only for the purpose of continuum detection. A peculiar continuum feature is the "bump" between $\lambda=4400 \AA$ and $\lambda=4500$ A. Donati-Falchi et al. (1984) also observed a continuum bump near this wavelength in their solar flare spectra, which for now remains unexplained.

The excess intensity in the Balmer jump region in this umbral kernel is of particular interest for modeling constraints. In Figure 9, we show the excess intensity at 15:09:30, 15:09:51, and 15:10:54 in the bluemost spectral region. The excess has little variation among the three times. As mentioned before, pristine focus is only achieved from $3654-3674 \AA$, which is indicated by the shaded region in Figure 9. Therefore, the slopes outside of this range cannot be characterized with high significance, due to the ambiguity from subtraction of the pre-flare (umbral) intensity level, which has a drastic spatial variation. Despite this, a broad continuum feature redward of the predicted Balmer edge at $\lambda=3646 \AA$ is well noticeable. The excess intensity extends from $\lambda \sim 3646 \AA$ while apparently increasing towards a broad maximum centered roughly at $\lambda \sim 3675 \AA$, which has an excess average intensity of $\sim 1.2 \times 10^{5} \mathrm{erg} \mathrm{s}^{-1} \mathrm{~cm}^{-2} \mathrm{sr}^{-1} \AA^{-1}$. We discuss this feature further in Section 5 in relation to previous studies. In the figure, the expected wavelengths of the higher order Balmer lines (H13-H19) are also indicated. We can identify Balmer lines in emission up to H14. In our spectra, an emission line is clearly located near the standard wavelength of H16 $(\lambda 3704)$, but He I $(\lambda 3705.0)$ and Fe I $(\lambda 3705.6)$ have been observed with just as large or larger flux as H16 in spectra of stellar flares Hawley \& Pettersen 1991; Fuhrmeister et al. 2008). 
Finally, we measure the ratio of excess continuum intensity in the bluest camera to the excess at the selected continuum regions at redder wavelengths at $\lambda=3915-3922$ and at $\lambda=4421-4451 \AA$ from Figure 6) for comparison to model values in a future paper. These spectral ranges are selected where focus is best and chromatic aberration does not affect the intensity. The ratios of excess continuum intensity at 15:09:30 are $\sim 0.6$ and 0.4, respectively.

\subsection{Flare contrast}

An additional parameter used to characterize white-light emission in flares is the flare contrast, or excess $\left\langle I_{\lambda}\right\rangle / I_{o}$ where $I_{o}=I_{\text {granulation }}$ is the non-flaring solar granulation intensity in Figure 3, To facilitate comparison with earlier spectra (e.g., Neidig 1983), we show in Figure 8 (square symbols, scale on the right axis) a measure of the flare contrast for the

four continuum windows from Figure 6 . The flare contrast is $\sim 10 \%$ throughout the spectral range, with slightly lower contrast of $\sim 5 \%$ in the far blue just redward of the Balmer edge wavelength. Note, some previous measurements of flare contrast allowed the subtraction of a nearby spectrum of the quiet sun at the same time as the flare. In our flare, the total intensity is low compared to granulation, so we must subtract the pre-flare umbral region to obtain a meaningful (positive) quantity. The flare contrast at 15:09:30 is also indicated in Figure 9. It exhibits a similar trend to the excess.

\section{Emission Line Analysis}

In addition to the significant continuum enhancement, several chromospheric emission lines are present in the flare spectra: $\mathrm{H} \gamma(\lambda 4341), \mathrm{H} \delta(\lambda 4101)$, Ca II H (blended with $\mathrm{H} \epsilon$,

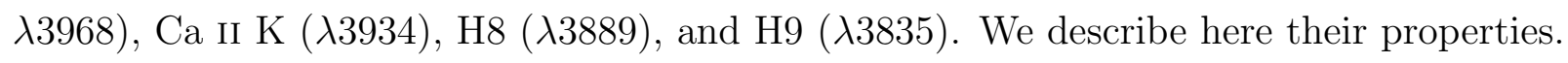




\subsection{Comparison with the Hard X Ray Fermi Light Curve}

In Figure 10 (a-e) we show the continuum-subtracted, line-integrated excess intensity as a function of time in $\mathrm{H} \gamma$ and $\mathrm{Ca}$ II $\mathrm{K}$ for the first slit position at the location of the umbral kernel (a), and in adjacent regions to the umbral kernel in the second, third, fourth, and fifth slit positions (b-e, respectively). The uncertainties of the integrated excess line intensity were calculated following the standard formula in the Appendix of Chalabaev \& Maillard (1983), which adds the uncertainty of the integrated continuum and line excesses in quadrature. Although the emission line excess extends over a larger region $\left(\sim 3^{\prime \prime}\right.$, Figure 4) compared to the continuum excess, we average the intensity only over the three brightest pixels along the slit. The emission line light curves are compared to the $15-21 \mathrm{keV}$ hard X-ray light curve obtained from Fermi/GBM and the 1-8 $\AA$ soft X-ray luminosity obtained from GOES. The same grey bars from Figure 6 indicate the times of significant continuum excess in panel (a). In the leftmost slit position of the raster (a), we see a general similarity in the normalized time variation of the hard X-rays and the optical lines. As we progress away from the leftmost slit position (panels b-e), we observe a more gradual response in the optical lines, yet reaching a comparable maximum value as in the first slit position. By the fifth slit position, i.e. $\sim 2^{\prime \prime} .5$ apart, the optical lines appear to evolve similarly to the soft X-ray emission. The gradual evolution in the chromospheric emission lines coincides with

the formation of new, low-brightness kernels at these later times, as seen in the development of the $\mathrm{H} \alpha+1.2 \AA$ umbral ribbon (Figure 5).

At the location of the umbral continuum excess, we find coincident peaks between the hard X-ray and the optical emission line light curves, but the 20 s cadence of the optical lightcurves makes it difficult to compare the precise timing with the much better sampled hard X-ray light curve $(\Delta t \sim 4 \mathrm{~s})$. The first enhancement at 15:08:27 in the hard X-rays corresponds well to the first impulsive enhancement in the optical lines, but we do not 
observe a significant continuum peak in Figure 6 at this time. The maximum of the hard X-rays at 15:09:25 corresponds to a major peak in both optical lines (15:09:30) and the continuum excess (15:09:30-15:09:51). The third emission line peak at 15:11:15 follows a significant excess continuum detection at 15:10:54 by one raster cycle (20 s) but does not readily have a corresponding peak in the hard X-rays. Rather, the continuum peak at 15:10:54 may be associated with a cotemporal peak in the $\mathrm{H} \gamma$ light curve at the same slit position but directly adjacent $\left(0^{\prime \prime} .4-1^{\prime \prime} .2\right)$ to the umbral WL kernel along the slit in the direction of the plage ribbon. This spatially adjacent flare enhancement (not shown in the figure) has two maxima in the $\mathrm{H} \gamma$ light curve at 15:09:30 - 15:09:51 and 15:10:54 with comparable values to the maxima at the position of the umbral WL kernel (Figure 10a). Interestingly, the second episode of continuum and line brightening at 15:10:54 is also not readily associated with a major, cotemporal hard X-ray peak. We note that a strong peak is also present in the $\mathrm{H} \gamma$ line in the second slit position at 15:10:54 (see Figure 10p).

The fourth major hard X-ray peak at 15:11:30 does not have a coincident peak in the optical emission lines originating from the umbral kernel and is probably associated with a different flaring area. Searching the flaring region we find a possible association with an optical line increase in the 19th and 20th slit positions in the plage flare ribbon. The first and second peaks of the Fermi light curve also correspond to peaks in the optical line emission originating from locations in the plage flare ribbon. An example light curve from a fixed spatial location (second white vertical line in Figure 1) in the plage flare ribbon is shown in Figure 11, which was obtained from the location of maximum optical line emission from the entire flare region. This light curve has a much more simple time evolution than the umbral kernel light curve (Figure 10a), but the line-integrated intensity is about twice as large even if the corresponding hard $\mathrm{X}$ ray burst is sensibly smaller than the following ones. However, the emission from the plage flare ribbon is not as spatially confined as the repeated optical line and $\mathrm{H} \alpha+1.2 \AA$ brightenings observed in the umbral kernel, which 
occur within a region confined to about 0 " .5 (Figure 5). The single-peaked light curve morphology is consistent with the relatively rapid plage flare ribbon progression towards the weak field region in Figure 1 .

For the umbral kernel, we compare the full-width at half-maximum (FWHM) of the light curves for $\mathrm{H} \gamma, \mathrm{Ca}$ II $\mathrm{K}$, and hard X-rays in Figure 10a. This measure gives a value known as $t_{1 / 2}$ which has been used for characterizing the timescales of continuum and line emission for flares on dMe stars (Kowalski et al. 2013). Considering the entire light curve duration, the timescale of the hard X-rays is longer than the timescale of the optical lines because the additional major X-ray peak at 15:11:30 occurs without an optical line counterpart at this spatial location. We calculate the newly-formed emission during the main peak at 15:09:30 by subtracting the flare emission at 15:08:50; from this, we find that the $t_{1 / 2}$ values are $20 \mathrm{~s}, 60 \mathrm{~s}$, and $60 \mathrm{~s}$ for the hard X-ray, H $\gamma$, and Ca II K light curves, respectively. Estimating the $t_{1 / 2}$ for the excess continuum in Figure 6 gives values ranging from 40-65 s, but this range is rather uncertain because the two significant continuum detections do not form a well-resolved light curve as for the emission lines. The significantly bright WL emission observed at 15:09:30 and 15:09:51 gives a lower limit of $20 \mathrm{~s}$ for the duration of the continuum excess, which is equal to the $t_{1 / 2}$ of the main hard X-ray peak. This timing information will be important for guiding modeling efforts (Section 5).

\subsection{The Balmer Decrement}

A broad wavelength coverage, intensity-calibrated spectrum allows the relative intensity to be measured in each emission line, giving the Balmer decrement, which is the ratio of the intensity of a particular Balmer line to that of another line (usually $\mathrm{H} \gamma$ ) in the series. Due to the paucity of data needed to determine this parameter, the Balmer decrement is used infrequently in solar flare studies, but it is a powerful constraint on temperature, electron 
density, and $\mathrm{H} \alpha$ optical depth (Drake \& Ulrich 1980), and can be used to test future radiative-hydrodynamic modeling of the flaring atmosphere. The results for the $\mathrm{H} \gamma$ Balmer decrement are shown for the solar flare in Figure 12, and compared with values observed in other solar and stellar environments.

We display decrements for the umbral kernel at 15:09:30 (the maximum in Figure 10a) and for the plage flare ribbon at 18th slit position at 15:08:44 (the maximum in Figure 11). We find that the $\mathrm{H} 8 / \mathrm{H} \gamma$ and $\mathrm{H} \delta / \mathrm{H} \gamma$ decrements are comparable in the umbral kernel and in the plage flare ribbon, but the $\mathrm{Ca}$ II $\mathrm{K} / \mathrm{H} \gamma$ decrement exhibits a significant variation between the two regions. In the umbral kernel, the $\mathrm{Ca}$ II $\mathrm{K} / \mathrm{H} \gamma$ decrement is less than 1 at 15:09:30 whereas in the plage flare ribbon, the decrement is greater than 1 . At the maximum at 15:11:15 in the umbral kernel (not shown), the Ca II K/H $\gamma$ decrement is 0.6( \pm 0.07$)$. The decrements from our C1.1 flare are compared to the early stage decrements from the M7.7 flare studied in Johns-Krull et al. (1997) and are found to be steeper.

Coincidentally, decrements from our $\mathrm{C} 1$ solar flare are very close to the decrements of a chromospherically active $\mathrm{M}$ dwarf (dMe) spectrum during quiescent times without any moderate or major flares. The decrements from various observed quiescent dMe spectra are shown in the right panel of Figure 12 (right panel). These decrements have been obtained from the literature including the dM3e star AD Leo from Kowalski et al. (2013) and other measurements of dMe stars reported in Hawley \& Pettersen (1991). The similarity between our C1.1 flare and the dMe stars is unambiguous for the of $\mathrm{H} 8 / \mathrm{H} \gamma$ and $\mathrm{H} \delta / \mathrm{H} \gamma$ decrements, whereas the $\mathrm{Ca}$ II $\mathrm{K} / \mathrm{H} \gamma$ decrements fall between the $\mathrm{C} 1.1$ solar flare plage ribbon and umbral kernel values. Note, the spectra of the dMe stars represent the irradiance from the entire visible hemisphere of the star, whereas the solar flare measurements represent only the emergent intensity at $\mu=0.74$. 


\subsection{Broadening of the Balmer Lines}

Symmetric broadening of hydrogen Balmer lines is thought to be at least partially due to the linear Stark effect from the ambient charge density in the flare chromosphere (Švestka 1963; Worden et al. 1984; Johns-Krull et al. 1997; Hawley \& Pettersen 1991). Stark broadening theory predicts larger energy shifts in the highest energy levels of hydrogen, so we examine the profiles of the highest order line with significant emission, H8 at $\lambda=3889$ A. We study the broadening at the same spatial locations and times as in Section 4.2 (at the light curve peaks of the umbral kernel and plage flare ribbion in Figures 10 a, 11 . respectively). The line profiles normalized to their peaks are shown in Figure 13 . We find the FWHM of H8 is between 1.5-1.7 $\AA$. The spectral resolution near H8 is at worst 1.3

$\AA$, which allows us to estimate an intrinsic FWHM of $\sim 1 \AA\left(\sigma_{\text {observed }}^{2}=\sigma_{\text {instr }}^{2}+\sigma_{\text {intrinsic }}^{2}\right.$ for a convolution of two gaussians). At the peak of the M7.7 flare reported in Johns-Krull et al. (1997), the FWHM of H8 was found to be $0.62 \AA$ or $\sim 50 \mathrm{~km} \mathrm{~s}^{-1}$, which is less than the velocity width of $\sim 80 \mathrm{~km} \mathrm{~s}^{-1}$ in our $\mathrm{C} 1$ flare. We also show the profiles of Ca II K at these same times and locations; this line is not affected by the linear Stark effect and generally has a profile that is close to the instrumental resolution, although there may be some broadening in the far wings. Comparing to the Ca II K broadening suggests that the H8 broadening is real, and a comparison of these two lines at 15:10:54 UT (not shown) in the umbral kernel does not reveal a significant difference. A meaningful understanding of the broadening mechanisms in our spectra requires accurate modeling of the Stark profiles in addition to the contributions from thermal and turbulent broadening, convolved with the instrumental profile. As discussed extensively by Johns-Krull et al. (1997), the various ways that Stark broadening is implemented in model codes can give somewhat ambiguous results for flare conditions; we will address this issue in a forthcoming modeling paper. 


\section{Summary and Discussion}

\subsection{Overview}

We have reported an optical continuum detection and the emission line characteristics during a small C1.1 flare, observed during a test run of a customized instrumental setup of the Horizontal Spectrograph on the Dunn Solar Telescope. The high spatial and temporal resolution of our observations allow us to clearly identify various portions of the flare that display different characteristics and evolution. In particular, within the flare ribbons, we detected a significant $(>4 \sigma)$ excess in optical continuum emission only in a tiny umbral kernel, with a diameter of about $0^{\prime \prime} .5$ (350 km, Figures 4 45). This umbral kernel exhibited repeated brightenings in the continuum and emission lines, with an evolution that is generally similar to the early impulsive phase $15-21 \mathrm{keV}$ hard X-rays detected by Fermi

but also with some noteable differences, including one major burst in the continuum excess (15:10:54) and optical lines (15:11:15) without cotemporal hard X-ray peaks (Figures 2, 4. 10.

No evidence of a significant continuum enhancement is found in any part of the plage flare ribbon, which develops concomitantly to the umbral one. However, several plage flaring kernels show enhanced chromospheric line emission in close temporal correlation with hard X ray peaks, with a rather impulsive character (e.g. Figure 11), which reflects the rapid spread of the plage ribbon within the weak magnetic field region.

\subsection{Balmer decrement and chromospheric line broadening}

Taking advantage of our broad spectral coverage, we calculated the Balmer decrements and line broadening both in the umbral kernel and at the location in the plage flare ribbon with the strongest line emission (Figures 12 , 13). We found the decrements to be 
steeper and the broadening to be greater than during a M7 flare reported in the literature (Johns-Krull et al. 1997). These differences may be the result of both better spatial resolution of our data and of the different flare phase considered, with our data reflecting the very early impulsive phase vs. the more gradual one of Johns-Krull et al. (1997).

We find an intriguing similarity of the Balmer decrements in our flare to those of other stellar environments, and speculate that there may be a similarity between the flaring conditions of a C1 solar flare and the lower atmospheric "quiescent" state of active M dwarf stars, which are known to show persistent hydrogen Balmer series and Ca II K line emission. Quiescent coronal soft X-ray emission from active stars has been shown to be consistent with a superposition of many individual flare events Güdel 1997; Kashyap et al. 2002; Güdel et al. 2003), and nonthermal turbulent broadening of quiescent transition region lines has been interpreted as evidence of transition region explosive events or microflaring events occuring in regions of magnetic flux emergence (Linsky \& Wood 1994). Persistent radio-emitting structures on dMe stars is indicative of the presence of nonthermal particles outside of major flaring events (Osten et al. 2006), and theoretical work indicates that particle acceleration and atmospheric heating is viable on active stars through a variety of mechanisms (Airapetian \& Holman 1998). Does the Balmer decrement suggest that a fraction of the quiescent dMe chromospheric emission level can be attributed to the superposition of events similar to long duration C1 solar flares occurring in several active regions simultaneously, and continuously, on the stars? We leave this question open for a future investigation.

The Hydrogen line intensities provided by the slab model of Drake \& Ulrich (1980) can be used to reproduce the Balmer decrement curve shown in Figure 12, and give a first indication of electron densities within the flaring region. By assuming a $T_{e} \sim 20,000 \mathrm{~K}$, we find that an $\mathrm{H} \alpha$ optical depth of $\tau \sim 100-400$ and a density $n_{e} \sim 10^{11.5}-10^{12} \mathrm{~cm}^{-3}$ 
are consistent with the measured $\mathrm{H} \delta / \mathrm{H} \gamma$ and $\mathrm{H} 8 / \mathrm{H} \gamma$ decrements. Neither a higher electron density, or a lower $\mathrm{H} \alpha$ optical depth can reproduce both decrements at once.

As mentioned in Section 4.3 , we plan to use these findings in a future work to estimate the effect of Stark broadening on the high order Hydrogen lines (H8 in particular), in comparison to the measurement of both Hydrogen and Ca II K profiles widths.

\subsection{Continuum excess: intensity and spectral distribution}

The value and spectral distribution of the continuum excess measured in the flaring umbral kernel can provide constraints on the heating mechanisms acting on the lower atmosphere. For example, a Balmer jump has been observed in several early flare spectra (Hiei 1982; Neidig 1983; Donati-Falchi et al. 1985; Neidig et al. 1994), which has led to the conclusion that the white-light continuum is comprised almost entirely of the Hydrogen recombination spectrum (as opposed to an enhanced photospheric continuum) with an origin in the upper chromosphere (Fletcher et al. 2007; Hudson 1972). This has found further support in very recent observations obtained with the Interface Region Imaging Spectrograph, that highlighted the presence of near-ultraviolet (2813 $\AA$ ) continuum enhancement in some flaring kernels, fully consistent with hydrogen recombination Balmer continuum emission (Heinzel \& Kleint 2014).

As reported in Section 2.4, the uncertainties introduced by chromatic aberration in our observations prevented an unambiguous determination of the detailed characteristics of the spectral slope at wavelengths in the Balmer continuum range $(\lambda<3646 \AA)$. However, we do note the apparent lack of a jump in excess intensity or flare contrast at $\lambda<3646 \AA$ relative to the intensity at redder wavelengths (Figure 9). A dominant optically thin 10,000 K spectrum would have produced a large Balmer jump ratio (i.e., the ratio of intensity at 
blue wavelengths to that at red wavelengths of $3646 \AA)$ that is $\sim 14$ Kunkel 1970; Neidig et al. 1993); we think that this should have been noticeable even with the data quality degraded due to chromatic aberration at $\lambda<3646 \AA$. It is possible that in our spectra the blending of Stark-broadened high-order Balmer lines 3 combined with the Stark broadening of the Balmer recombination edge could smear the Balmer jump making it non-detectable. Still, we note that other white light flares reported in the literature either did not display a Balmer jump, or had other properties not readily explained by a Hydrogen recombination spectrum. In particular, a strong blue continuum emission at $\lambda<4000 \AA$ has been often reported (Hiei 1982; Neidig \& Wiborg 1984), and shown by Donati-Falchi et al. (1985) as the result of the blending of Stark-broadened high-order Balmer lines in a dense chromosphere. In the model of Donati-Falchi et al. (1985), this "bump" in the blue continuum peaks at $\lambda \sim 3675 \AA$ and becomes more prominent and shifts to redder wavelengths as electron density in the flare region increases. Existing models of Stark broadening imply that such blue "continuum" emission originates from a location with electron density in excess of $10^{13} \mathrm{~cm}^{-3}$ and electron temperature between 7,000 and 10,000 K. Interestingly, in our excess spectra we observe a relatively featureless, broad bump peaking at $\lambda \sim 3675 \AA$ (Figure 9). Taken at face value, the electron densities inferred from interpreting this feature via Stark broadening appear at odd with those derived in Sect. 5.2, unless more optically thin features such as the higher order Balmer lines probe different portions of the flaring atmosphere. However, additional spectra that are not affected by chromatic aberration will be needed to confirm and understand this feature.

Following Kerr \& Fletcher (2014), we also compare our spectral data to a blackbody

\footnotetext{
${ }^{3}$ The spectral region from 3654-3674 Å contains the rapidly converging hydrogen Balmer lines H23 through at least H40; the center wavelengths of H23 and H24 are separated by $\sim 2.5 \AA$ whereas H39 and H40 are separated by only $0.5 \AA$.
} 
spectrum representing a photospheric flare continuum. This, however, requires a wellresolved measurement of the intensity. Although the umbral kernel is unresolved in the spectra, the resolved area from IBIS $\mathrm{H} \alpha+1.2 \AA$ gives an actual spatial extent $\left(0^{\prime \prime} .5\right)$ of the kernel that is not far below the resolution of the spectra $\left(0^{\prime \prime} .67 \times 0.8^{\prime \prime}\right)$; therefore, we can give a lower limit on the radiation brightness temperature assuming a blackbody intensity. The maximum excess + pre-flare intensity from $\lambda=4421-4451 \AA$ in the umbral kernel at 15:09:30 gives a brightness temperature of $T_{\text {rad }} \sim 5400 \mathrm{~K}$ for the flare, compared to $T_{\text {rad }} \sim 5200 \mathrm{~K}$ for the pre-flare umbra. This flare radiation temperature is similar to the optical color and brightness temperatures found in Kerr \& Fletcher (2014) for an X-class flare and by Watanabe et al. (2013). A photospheric temperature increase of only $200 \mathrm{~K}$ (also similar to that found in Kerr \& Fletcher (2014)) likely implies a flare continuum emissivity dominated by $\mathrm{H}^{-}$emission processes (recombination and bremsstrahlung). We note that this increase is far below that implied by a blackbody color temperature of $\sim 9000 \mathrm{~K}$, recently found in the Sun-as-a-Star, superposed epoch analysis of C class flares from Kretzschmar (2011). The ratio of intensity at $\lambda=3914-3922 \AA$ to the intensity at $\lambda=4421-4451 \AA$ gives a color temperature (Section 3) in the umbral kernel for our data, but we do not expect the flare spectrum between these wavelengths to exhibit a Planckian shape for a small temperature increase of $200 \mathrm{~K}$ implied by the brightness temperature, due to similar complicated opacity effects that produce the pre-flare umbral spectrum. There are relics of the background spectrum in the excess spectrum at the $\mathrm{Ca}$ II $\mathrm{H}$ and $\mathrm{K}$ absorption wings and at the G-band at $\sim 4300 \AA$ in Figure 8. These features may help constrain the origin of the emission using models that include wavelength-dependent opacities.

We finally turn to the flare contrast at different wavelengths, as defined in Section 3.3. This quantity can be largely affected by the spatial resolution, as discussed by Jess et al. (2008) for a C2.0 flare. The flare contrast in Figure 8 was found to be $\sim 10 \%$, which is the average excess intensity relative to a nearby non-flaring (granulation) region away 
from the spot and between plage regions. Compared to some larger flares with spectra in the literature (Figure 3 of Neidig 1983), the optical contrast values near $\lambda \sim 3920 \AA$ are quite similar, but the contrast at the bluest wavelengths is significantly smaller. It should be noted, however, that these older spectra typically did not sample the brightest kernels. If instead we calculate the flare contrast relative to the pre-flare umbral intensity (Section 3, $I_{o}=I_{\text {umbra }}$ ), we obtain values of $\sim 20 \%$ or more for our flare. However, the true spatial extent of the umbral kernel is only $A_{\text {IBIS }} \sim 10^{15} \mathrm{~cm}^{2}$ (Section 3.1) compared to the unresolved area from the spectra: $A_{\text {spec }}=0 .{ }^{\prime \prime} 67 \times 0 .{ }^{\prime \prime} 8 \sim 3 \times 10^{15} \mathrm{~cm}^{2}$. This allows us to provide an estimate of the actual values of the flare contrast to be $\gtrsim 30 \%(\gtrsim 60 \%$ relative to the pre-flare umbral intensity). This adjusted value of the flare contrast (at $\lambda=3914-3922 \AA)$ is similar to the flare contrast value relative to the nearby granulation

derived from a spatially resolved observation at $\lambda \sim 3954 \AA$ for the C2.0 flare in Jess et al. (2008). The adjusted value of the flare contrast relative to pre-flare background is even consistent with the values obtained in several bright kernels at $\lambda=4275 \AA$ during the much larger X13 flare of 24-Apr-1984 (Neidig et al. 1994). In making these adjustments we have multiplied by a factor of $A_{\text {spec }} / A_{\text {IBIS }} \sim 3$, which is consistent with summing the excess spectral intensity over the three spatial pixels (instead of averaging to produce $\left.\left\langle I_{\lambda}\right\rangle\right)$. Applying the areal adjustment to the calculation of brightness temperature from $\lambda=4421-4451 \AA$ gives an increase of only $500 \mathrm{~K}$ (compared to an increase of $200 \mathrm{~K}$ without the adjustment), to a value of $T_{\mathrm{rad}} \sim 5700 \mathrm{~K}$.

\subsection{RHD modeling}

Detailed radiative-hydrodynamic (RHD) models of flares have been employed in the last years for a more rigorous interpretation of flare spectra. One such example is the RADYN code (e.g., Carlsson \& Stein 1994, 1995, 1997), which has been modified to 
incorporate flare energy deposition (Hawley \& Fisher 1994; Abbett \& Hawley 1999; Allred et al. 2005). The atmospheric dynamics (Fisher 1989) and optical continuum properties Cheng et al. 2010) depend strongly on the preflare atmospheric state (e.g., umbral vs. granulation), viewing angle $(\mu)$, and parameters of the nonthermal electron spectrum (low-energy cutoff, $E_{c}$, power-law index, $\delta$, and energy flux) which is usually assumed to power the chromospheric emission.

Although the existing RHD models (Abbett \& Hawley 1999; Allred et al. 2005; Cheng et al. 2010) present results for generalized combinations of heating parameters and preflare atmospheric states, they can provide some important insight also for the case analyzed in this paper. The models of Cheng et al. (2010) consider the flare contrast at several optical and infrared wavelengths over a large parameter space of the nonthermal electron spectrum (used as the heating mechanism) while employing a model umbra for the preflare atmosphere, which would be most appropriate for our flare. The model with $E_{c}=20$ $\mathrm{keV}, \delta=5$, and nonthermal electron energy flux of $10^{10} \mathrm{erg}_{\mathrm{cm}}^{-2} \mathrm{~s}^{-1}(\mathrm{~F} 10)$, at $\mu=0.95$, produces a contrast of only $3 \%$ at $4300 \AA$ after 18 s of constant heating, which is far below the observed contrast of $20 \%$ (and the inferred corrected contrast of $\gtrsim 60 \%$ ) in our flare. A larger beam flux $\left(10^{11} \mathrm{erg}_{\mathrm{cm}}^{-2} \mathrm{~s}^{-1}, \mathrm{~F} 11\right)$, higher low-energy cutoff (40 keV), and flatter spectral index $(\delta=3)$ produce an acceptable value of the contrast at our viewing angle with a temperature increase of $300 \mathrm{~K}$ in the upper photosphere from chromospheric radiative-backwarming. The models of Allred et al. (2005) produce an optical contrast (at $\lambda=5000 \AA$ ) of $30 \%$ for an F11 simulation relative to the granulation intensity, which is consistent with our inferred value (at $\lambda=4450 \AA$ ) relative to granulation. However, a large beam flux and a moderate to high $(20-40 \mathrm{keV})$ low-energy cutoff would have produced also a large amount of Balmer continuum emission, and resulted in contrast values of $\sim 50-230 \%$ relative to granulation at wavelengths just blueward of the Balmer edge (Abbett \& Hawley 1999; Allred et al. 2005). The lack of a strong Balmer continuum 
component (Section 3 and Figure 9) in our spectrum make it unlikely that such powerful level of energy deposition in the upper chromosphere and subsequent radiative-backwarming of the upper photosphere can explain the observations.

\subsection{Future work}

The preliminary analysis performed in the previous sections suggests that the observed properties of our flare are difficult to reconcile with simpler, static models, or with existing grids of RHD models. We thus plan to undertake detailed radiative-hydrodynamic models of this particular flare, utilizing our comprehensive set of observables to constrain the simulation.

The Fermi hard X ray data will be utilized to derive an energy spectrum of nonthermal electrons for input to the models. From our optical spectral data we can also provide estimates for the time profile of heating, and a lower limit of the heating flux necessary to sustain the excess optical flare emission. The observed timescale $\left(t_{1 / 2} \sim 20 \mathrm{~s}\right.$ and duration of $40 \mathrm{~s}$ ) of the main hard X-ray burst can be used to guide the duration of the energy deposition time-profile for modeling the first significant continuum enhancement. Using a simplifying, crude assumption that $\left\langle I_{\lambda, \mu}\right\rangle$ is isotropi 4 , $F_{o p t}=\pi \int\left\langle I_{\lambda, \mu=0.74, \text { excess }}\right\rangle d \lambda$, integrated over the wavelengths of our observations, we obtain at the maximum of the umbral kernel (at 15:09:30), $F_{\text {opt }} \sim 5 \times 10^{8} \mathrm{erg}^{-2} \mathrm{~s}^{-1}$. Adjusting this value by $A_{\text {spec }} / A_{\text {IBIS }}$ suggests the radiative flux to be at least $1.5 \times 10^{9} \mathrm{erg} \mathrm{cm}^{-2} \mathrm{~s}^{-1}$, which is still a lower limit because our spectrum has a limited wavelength coverage and does not include radiation emitted in the UV, red optical, and infrared. Given the energy constraints from our data, future RHD models that aim to reproduce the optical emission during the main X-ray peak

\footnotetext{
${ }^{4}$ In the optically thin, plane-parallel approximation, $F_{\text {opt }}=2 \pi \times 0.74 \int\left\langle I_{\lambda, \mu=0.74 \text {,excess }}\right\rangle d \lambda$
} 
should carefully explore a range of nonthermal electron energy fluxes around the value of $\sim 10^{9} \mathrm{erg}_{\mathrm{cm}}^{-2} \mathrm{~s}^{-1}$, which is well feasible with the current generation of simulations Abbett \& Hawley 1999; Cheng et al. 2010). The models of Abbett \& Hawley (1999) and Cheng et al. (2010) do not produce a significant optical contrast with such a low beam flux, but the particular combination of modeling parameters in these studies may not be appropriate for our flare.

Alternative heating mechanisms may be needed to explain the second continuum enhancement at 15:11 without an obvious, cotemporal X-ray peak. If there is a relationship to the main X-ray peak, a delay of $90 \mathrm{~s}$ appears slightly too long for the lifetime of a downward-directed heated compression wave, or an Alfvénic disturbance, to reach the conditions of optical line and continuum formation (Fisher 1989; Russell \& Fletcher 2013). Models of stochastic acceleration in magnetized turbulence predict that the relative amount of proton to electron acceleration increases in environments with a denser plasma, longer magnetic loops, or a weaker magnetic field (Petrosian \& Liu 2004; Emslie et al. 2004), all of which may pertain to the atmospheric conditions during episodes of magnetic reconnection in the late impulsive phase.

\section{Conclusions}

We observed a small-amplitude, long duration GOES C1.1 flare, and the observed and inferred optical properties (contrast and brightness temperature) appear similar to some X-class flares. Multiplying the area of the white-light kernel in our C1.1 flare by $F_{\text {opt }}$ gives a

power of $L_{\mathrm{opt}}=1.5 \times 10^{24} \mathrm{erg} \mathrm{s}^{-1}$, which is comparable to the $1-8 \AA$ soft X-ray luminosity of the entire flare region (Figure 2). Indeed, the soft X-ray emission in solar flares is only a minor fraction (1-10\%) of the total radiated energy Kretzschmar 2011; Emslie et al. 2012), and can vary largely from event to event (Neidig \& Kane 1993). What aspect of 
the flare energy release can explain such a variation in the soft X-ray response, and also in the apparent amount of Balmer continuum emission, while producing similar properties at optical wavelengths?

The broad spectral coverage of our data, in particular the rarely-observed blue wavelength range around the Balmer edge, provides an opportunity to confront the evolution of our flare with results from modern RHD models. Very few events have been observed so comprehensively, providing a rigorous way to guide the models and assess their assumptions and results. For example, the hard X ray clearly informs what kind of beam we can use, and for how long energy deposition is sustained. The resolved area of the WL kernel constrains the heating flux, which is important for determining the flare dynamics and the contrast at wavelengths where Balmer continuum emission is expected. Furthermore, the WL kernel is observed from the very beginning of the flare, so we can reliably compare the results of the models with spectra at the appropriate time. Most observations of WL flares in the past were never observed in the very impulsive phase, and rarely did spectral observations sample the brightest kernels. We can investigate the different properties of umbral and plage kernels that develop concomitantly to address why one develops WL and the other does not while producing much stronger chromospheric line emission. The connection among particle acceleration (or other heating mechanisms) and the magnetic and atmospheric environment of the flare will likely be necessary to explore with detailed modeling, in order to explain the range of WL properties in the bluest wavelengths around the Balmer jump.

We want to thank the DST observers, D. Gilliam, M. Bradford, and J. Elrod, for their precious and patient assistance. The authors thank Suzanne Hawley for useful discussions on the importance of obtaining solar spectra with broad wavelength coverage, K. Tolbert for assistance obtaining the Fermi data, M. Janvier for helpful suggestions and discussions, 
and an anonymous referee for helpful constructive comments. The authors are pleased to acknowledge the support of the International Space Sciences Institute (ISSI) in Bern. IBIS is a project of INAF/OAA with additional contributions from University of Florence and Rome and NSO. The National Solar Observatory is operated by the Association of Universities for Research in Astronomy, Inc., under a cooperative agreement with the National Science Foundation. This research was supported by an appointment to the NASA Postdoctoral Program at the Goddard Space Flight Center, administered by Oak Ridge Associated Universities through a contract with NASA. The research has received funding from the European Community's Seventh Framework Programme (FP7/2007-2013) under agreement \#606862 (F-CHROMA). AFK acknowledges the National Solar Observatory for travel support. LF acknowledges support from STFC grant ST/L000741/1. 

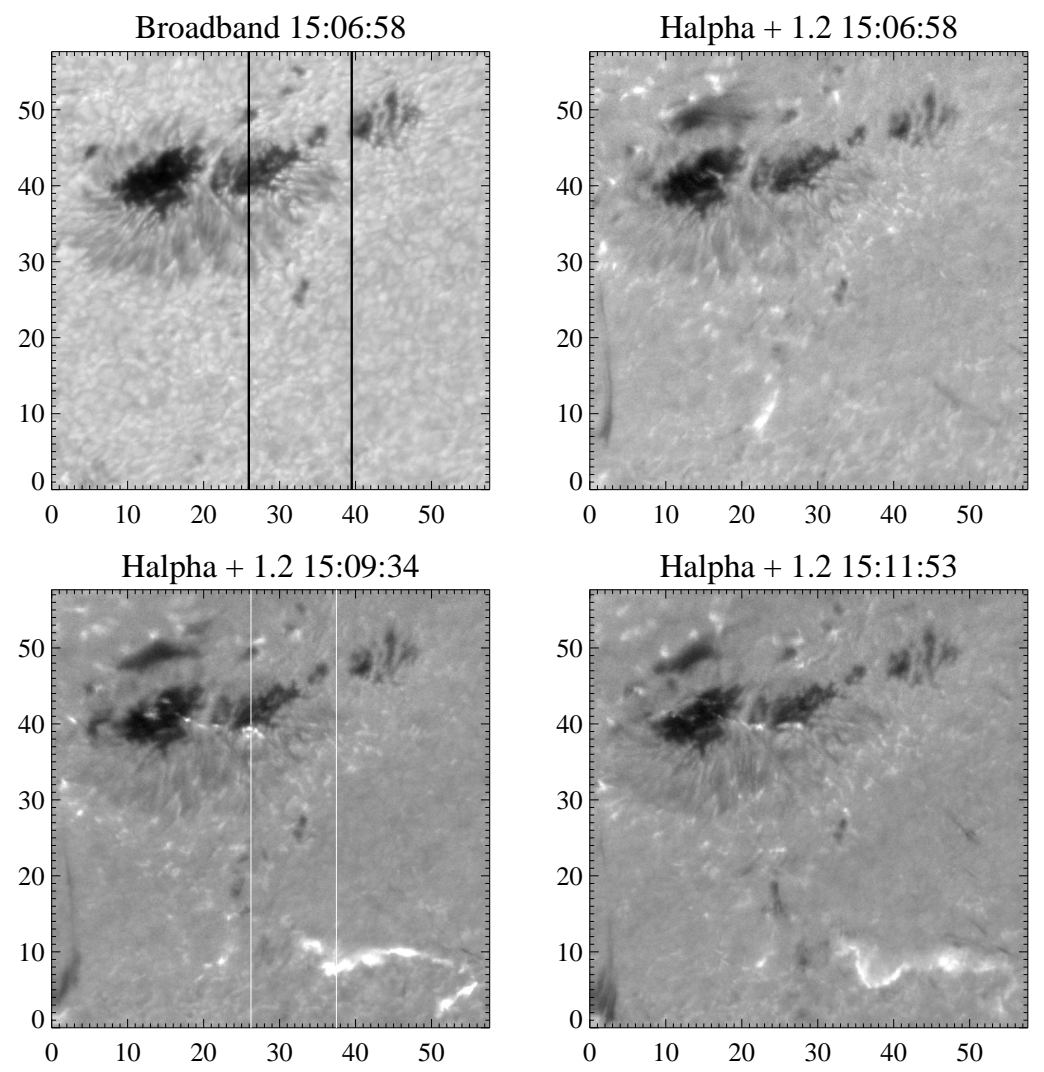

Fig. 1.- Abridged caption: Central portion of the IBIS field of view. Axes are in units of arcseconds. Top left: Pre-flare broadband image at $6360 \AA$. The two vertical black lines outline the edges of the HSG raster. Top right: $\mathrm{H} \alpha+1.2 \AA$, at the same preflare time. An early brightening is already visible within the larger spot. Bottom left: $\mathrm{H} \alpha+1.2 \AA$ near the time of largest hard X-ray peak in the Fermi curve. The left white line indicates the HSG slit position at 15:09:30 and the right white line indicates the HSG slit position at 15:08:44. Excess continuum was detected in the small flare kernel crossed by the slit around position $\left(26^{\prime \prime}, 39^{\prime \prime}\right)$ at this time. Bottom right: $\mathrm{H} \alpha+1.2 \AA$ at a later time during the flare development. Note the motion of the plage flare ribbon away from the earlier position. The images maintain the native orientation, with vertical direction along the parallactic angle; the east limb direction is roughly towards the bottom of the figure and north is to the right. 


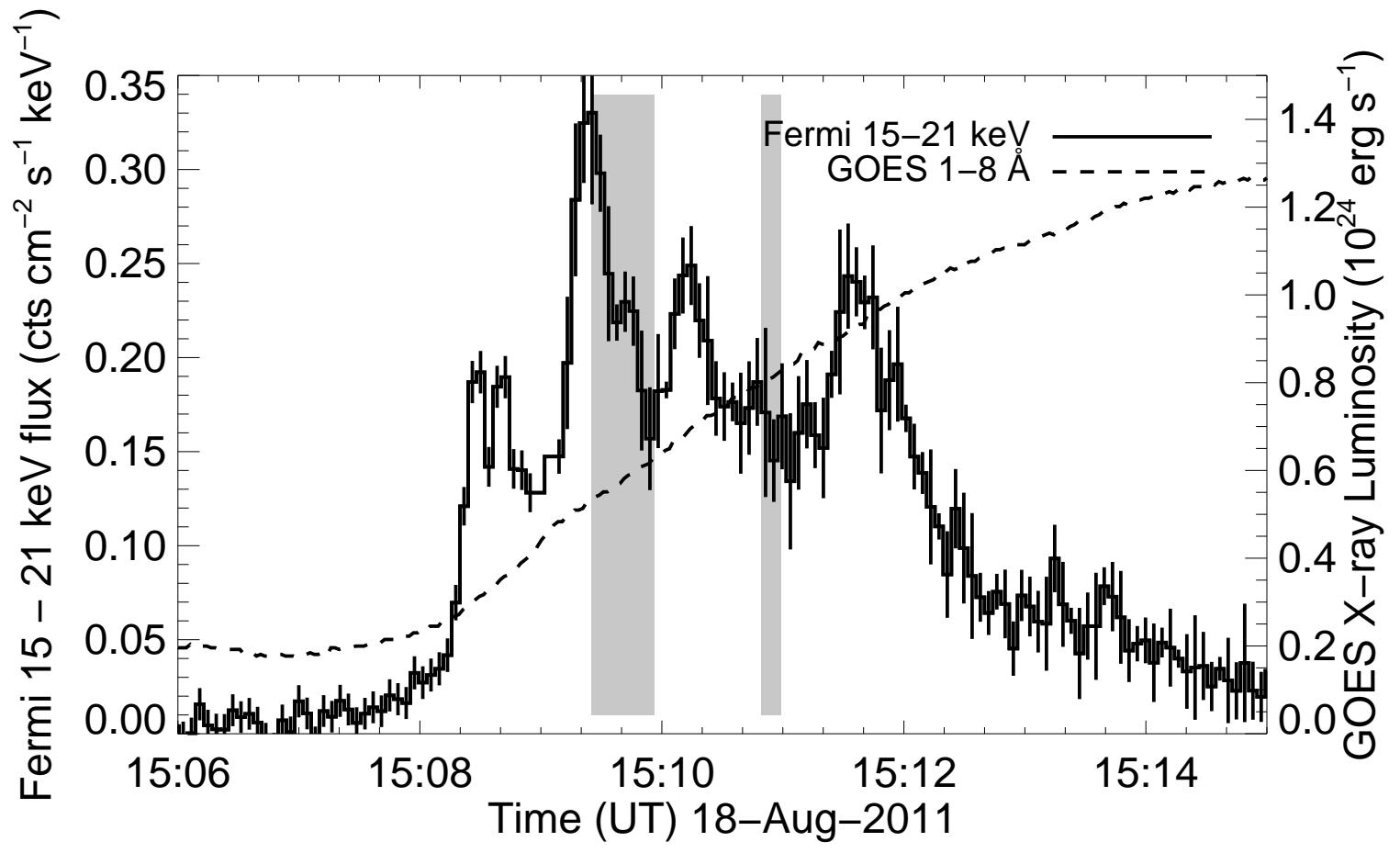

Fig. 2.- The Fermi $15-21 \mathrm{keV}$ hard X-ray count flux light curve (left axis) shown with the GOES 1-8 A luminosity (right axis) of the C1.1 flare SOL2011-08-18T15:15 from AR 11271. The timing of the simultaneous $\mathrm{H} \alpha+1.2 \AA$ and optical continuum enhancements are indicated by vertical grey bars. Each Fermi data point has a live time of $4.07 \mathrm{~s}$. 


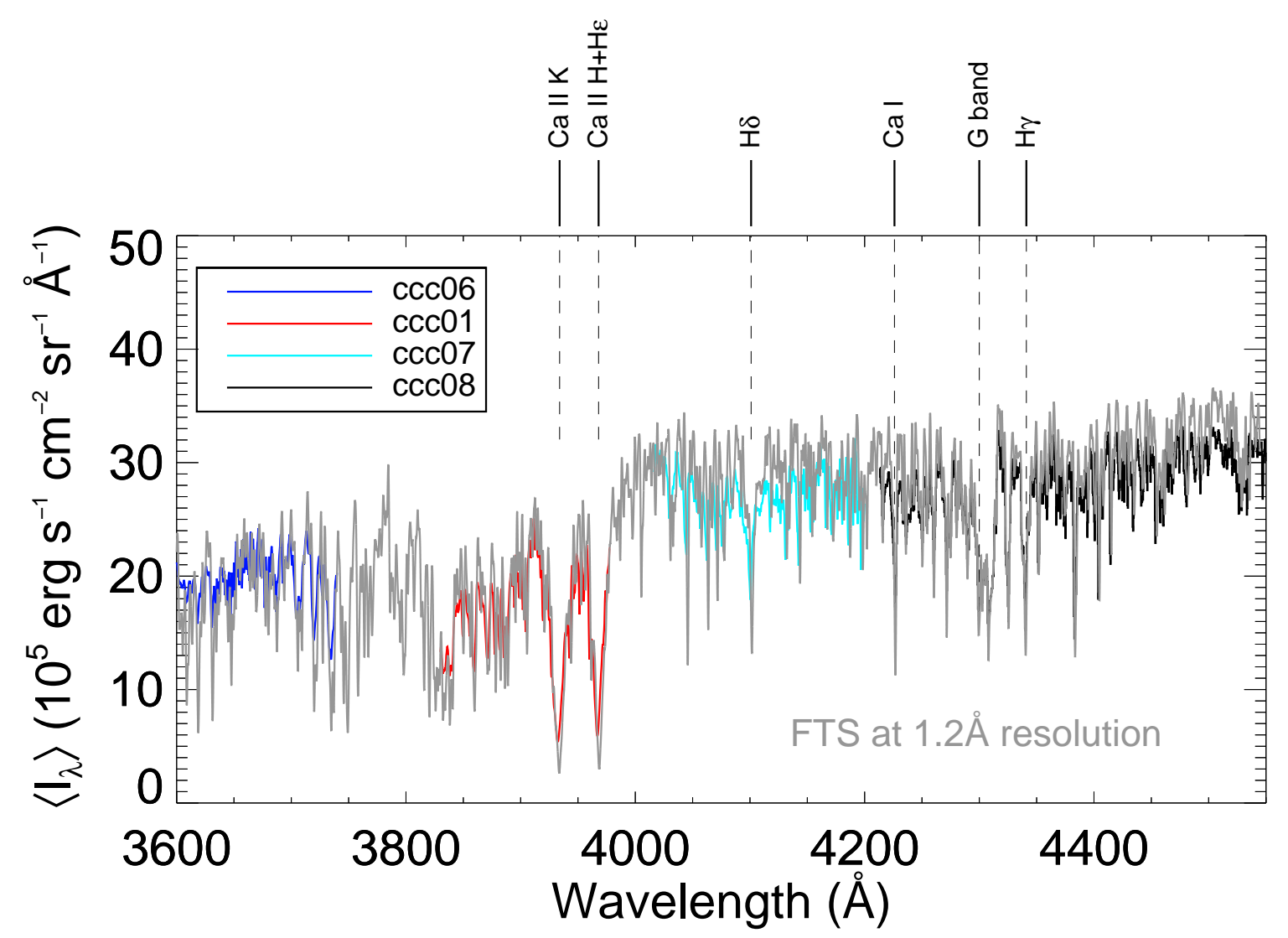

Fig. 3.- Total intensity (averaged over 3 spatial pixels; $\left\langle I_{\lambda}\right\rangle$ ) of a non-flaring granulation region away from plage and umbra at the 18th slit position at 15:07:20, compared to the FTS disk-center intensity adjusted by the limb darkening at $\mu=0.74$ and convolved with a Gaussian of FWHM=1.2 A. The intensity level and shape of the observed solar continuum is reproduced well in this quiescent region. 

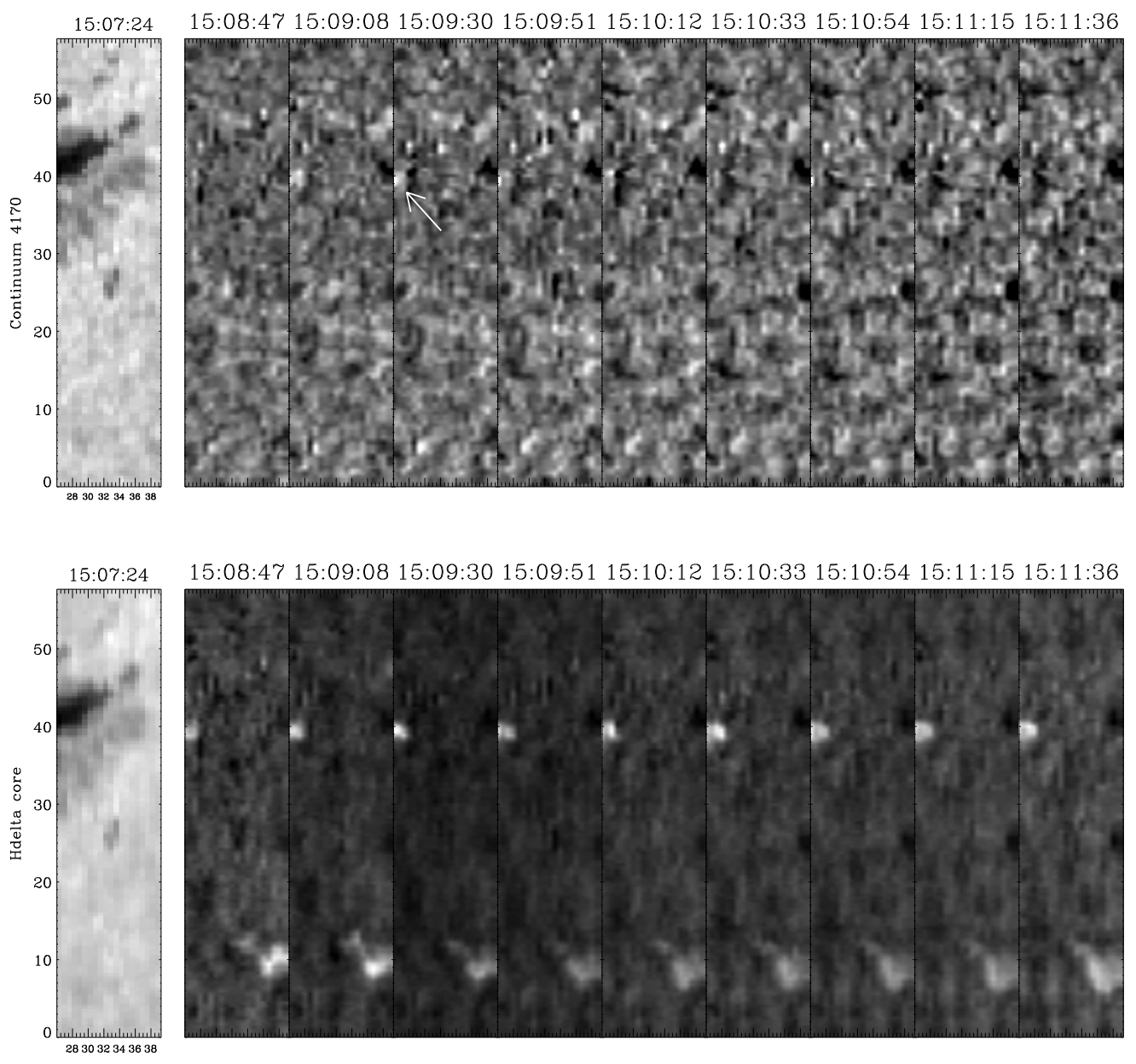

Fig. 4.- Top panels: HSG raster continuum image in the pre-flare (left panel), and images of the continuum ratio (enhancement) at $4170 \AA$ at various times during the flare development. The enhancement images are scaled between $-10 \%$ and $+15 \%$ of the pre-flare values in the same spatial positions. The white arrow indicates the WL enhancement discussed in the text. Bottom panels: the same as top panels, for the $\mathrm{H} \delta$ line core, scaled between $-30 \%$ and $+40 \%$. Note the sharper definition of the continuum image, highlighting photospheric features, and the larger extension of the flare kernels as imaged by the chromospheric line core emission. The time indicated above the panels refers to the beginning of each raster scan, which proceeds from left to right in the images. 


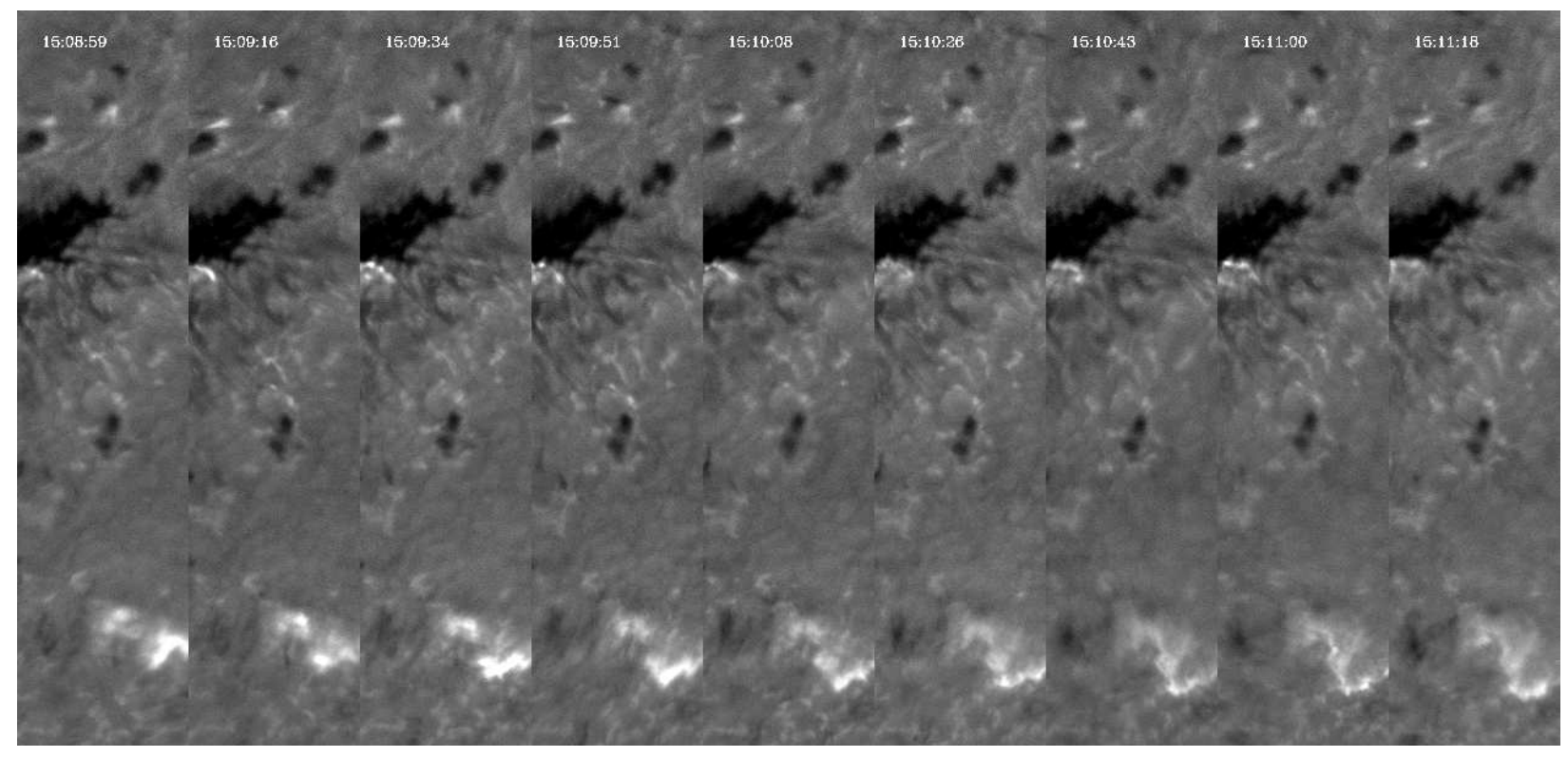

Fig. 5.- IBIS data showing the $\mathrm{H} \alpha+1.2 \AA$ wing evolution for the approximate field of view covered by the HSG spectral raster. 


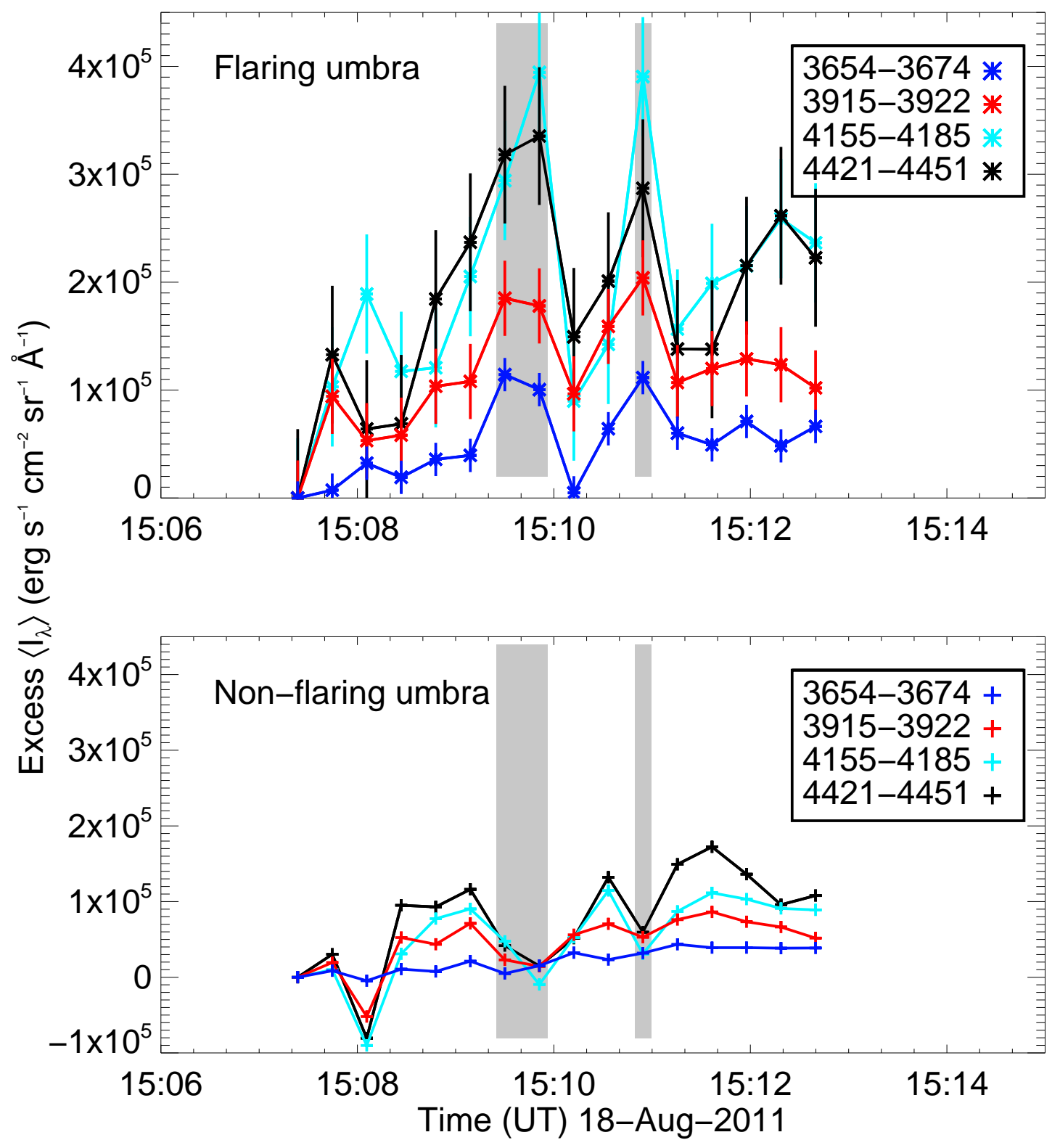

Fig. 6.- Top: The time-evolution of the excess continuum intensity in four spectral regions, extracted from the umbral kernel. The vertical grey bars indicate the times of the significant flare continuum detections. Bottom: The excess continuum variations in a nearby non-flaring region of the umbra. The standard deviation of this panel gives the statistical error in the top panel light curve. 

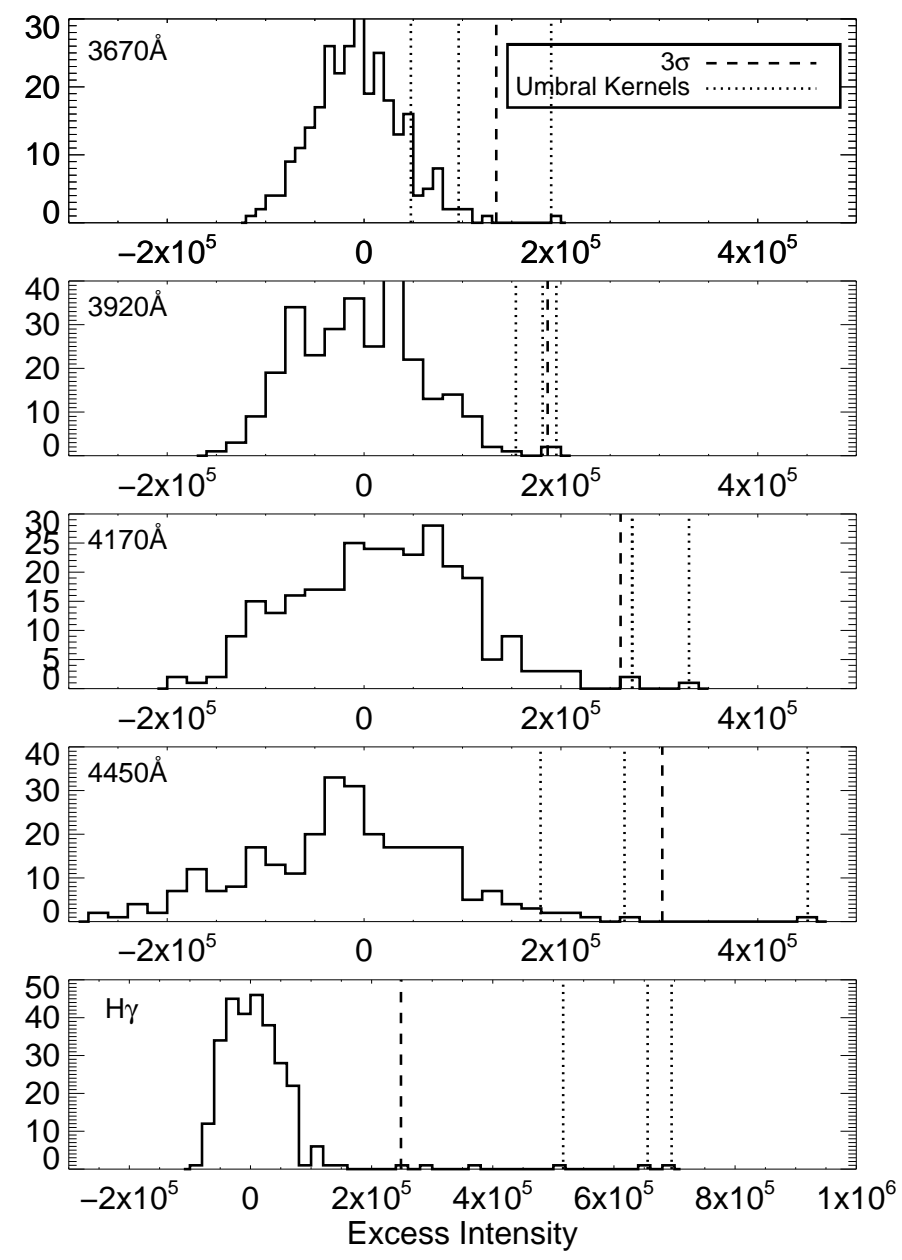

Fig. 7.- Histograms of the excess intensity (subtracting 15:07:24 from 15:09:30) over a spatial cut through the HSG data, at selected continuum intervals and $\mathrm{H} \gamma$. The value corresponding to $3 \sigma$ of the distribution is indicated by dashed lines, whereas the values for the 3 pixels averaged to obtain the excess $\left\langle I_{\lambda}\right\rangle$ for the umbral kernel are indicated by dotted lines. Note that the value of $\sigma$ in this figure represents the spatial variation of the excess, whereas the value of $\sigma$ in Figure 6 describes the temporal variation of the excess. 


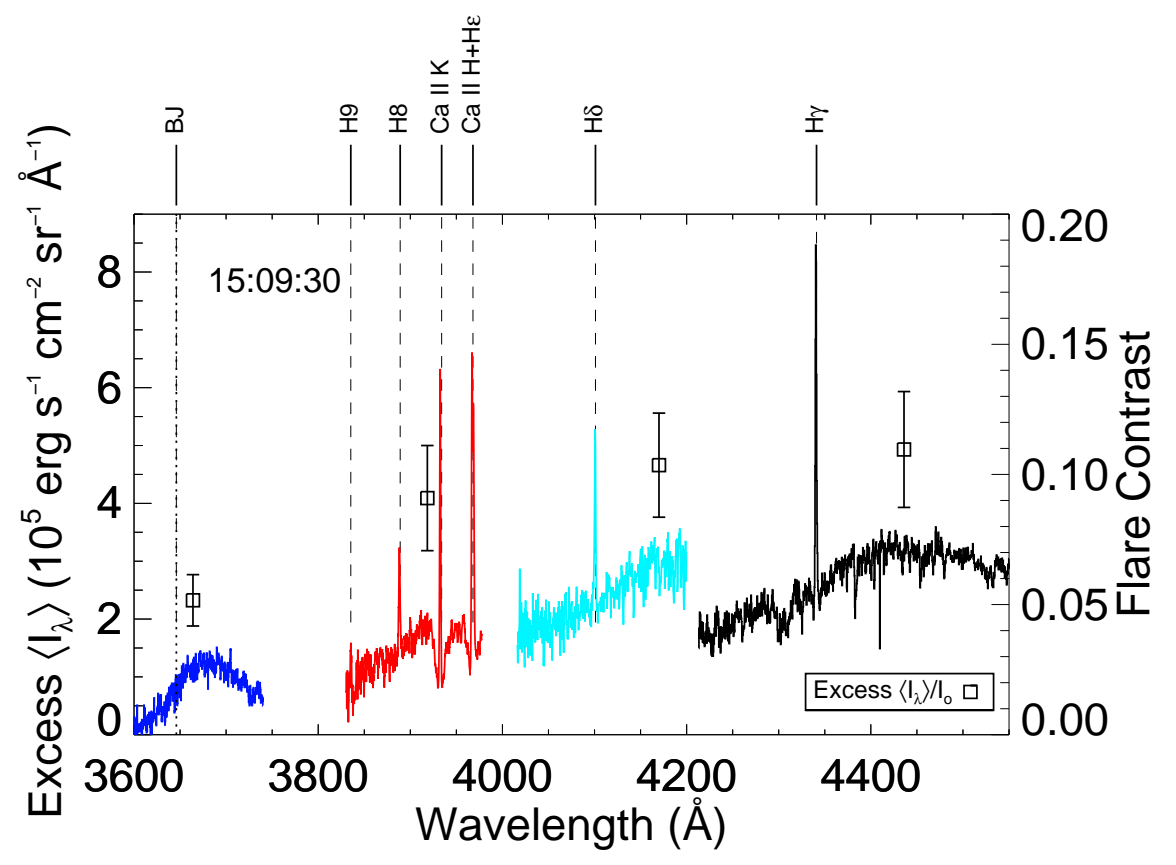

Fig. 8. - The full spectral range of the excess intensity at 15:09:30 in the umbral kernel at the first slit position in the spectral raster. The intensity is averaged over three spatial pixels. The right axis (square symbols) show the flare contrast in selected continuum wavelength regions. 


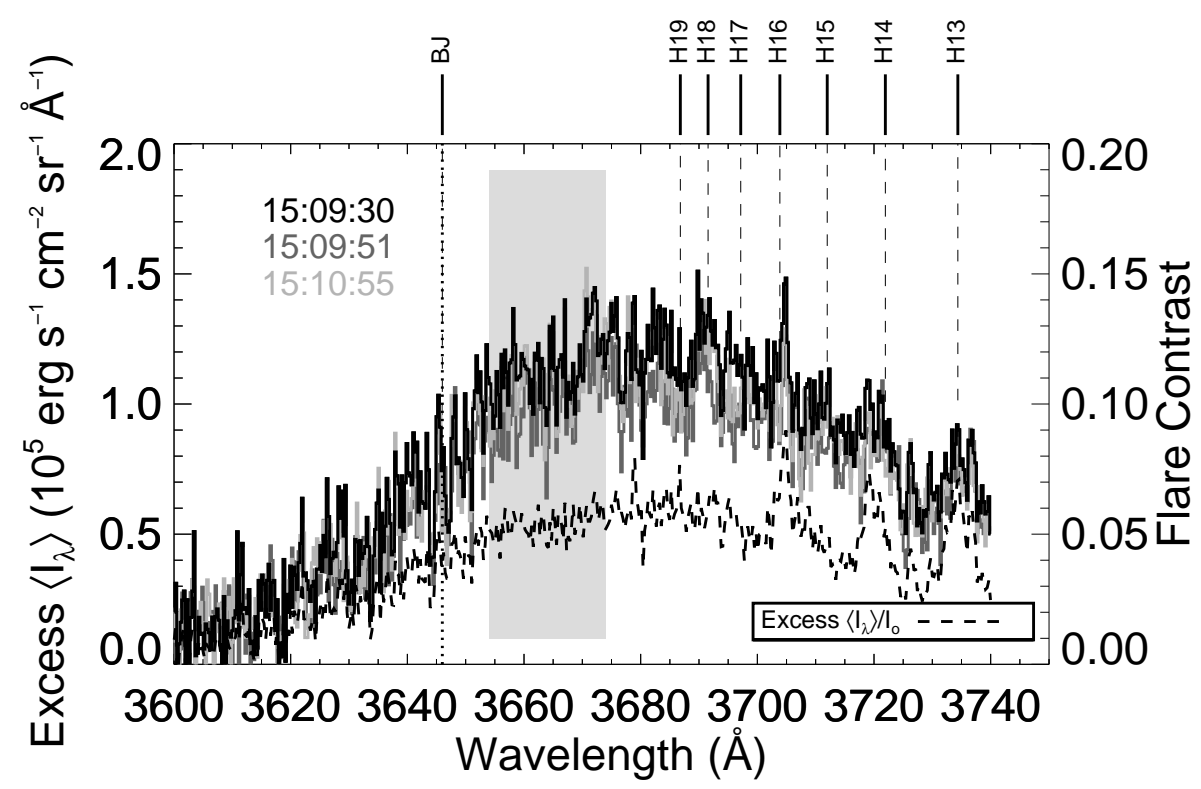

Fig. 9.- The excess intensity in the umbral kernel at the first slit position in the spectral raster, shown for the bluemost spectral region at three times. The expected wavelengths of the Balmer jump (BJ) and the higher order Balmer lines are indicated; H14 is the last visible Balmer line, and the feature near the wavelength of H16 could be a blend of Fe I and He I at this wavelength (see text). The shaded area indicates the wavelength region not affected by chromatic aberration. The right axis (dashed line) shows the flare contrast. 

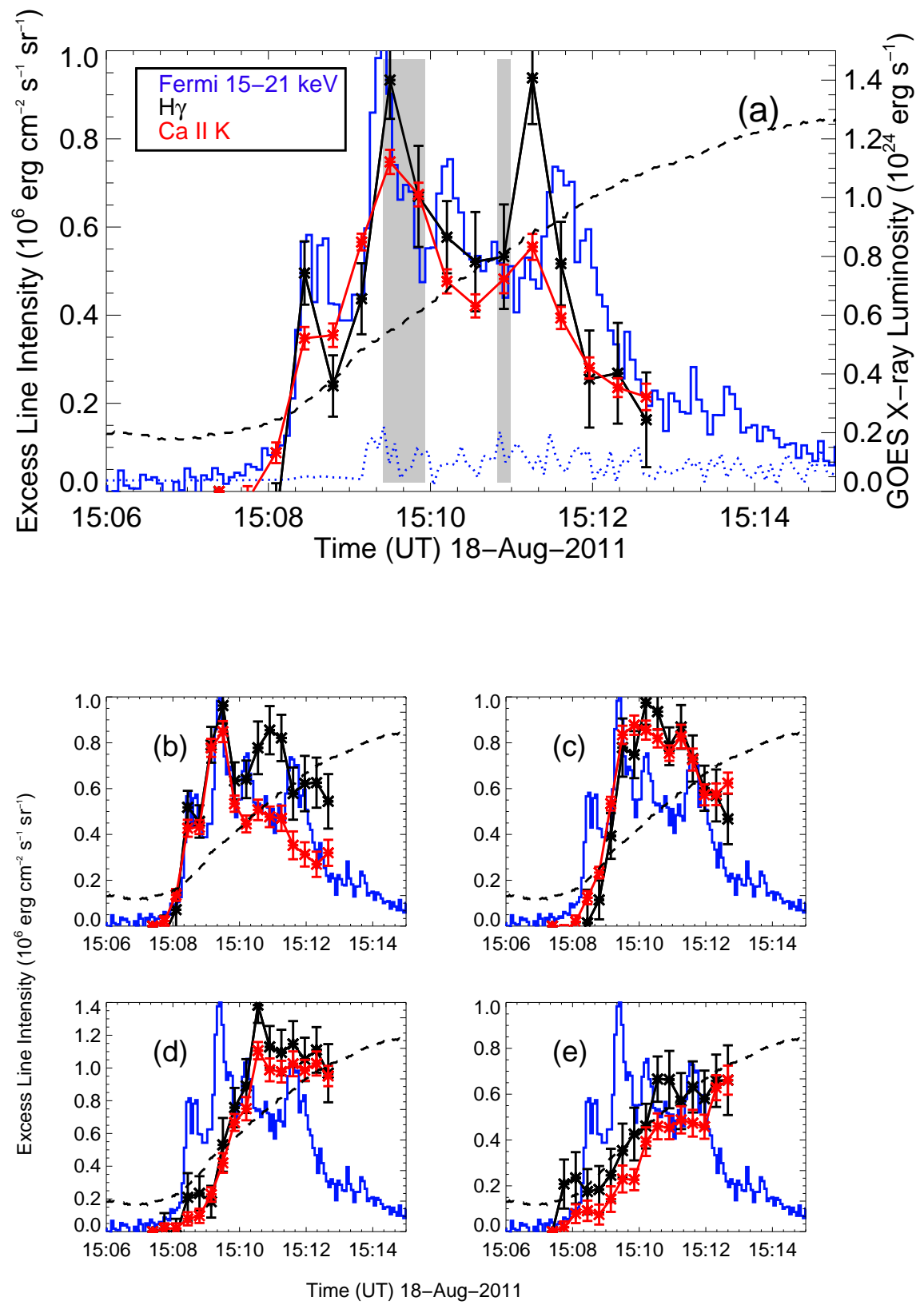

Fig. 10.- (a) The light curves of $\mathrm{Ca}$ II $\mathrm{K}$ and $\mathrm{H} \gamma$ emission lines from the umbral kernel compared to the Fermi/GBM data from 15-21 keV (normalized to the peak value of 0.33 counts $\mathrm{cm}^{-2} \mathrm{~s}^{-1} \mathrm{keV}^{-1}$ ). The average excess line intensity over three spatial pixels is shown. The dashed line is the GOES 1-8 A luminosity (right axis), and the dotted line is the $1 \sigma$ error for the Fermi data. Grey vertical bars indicate the times of a significant continuum excess. Panels (b)-(e) show the same quantities for the regions adjacent to the umbral kernel, in the second, third, fourth, and fifth slit positions, respectively. Note the rescaling of the y-axis in panel $(d)$. 


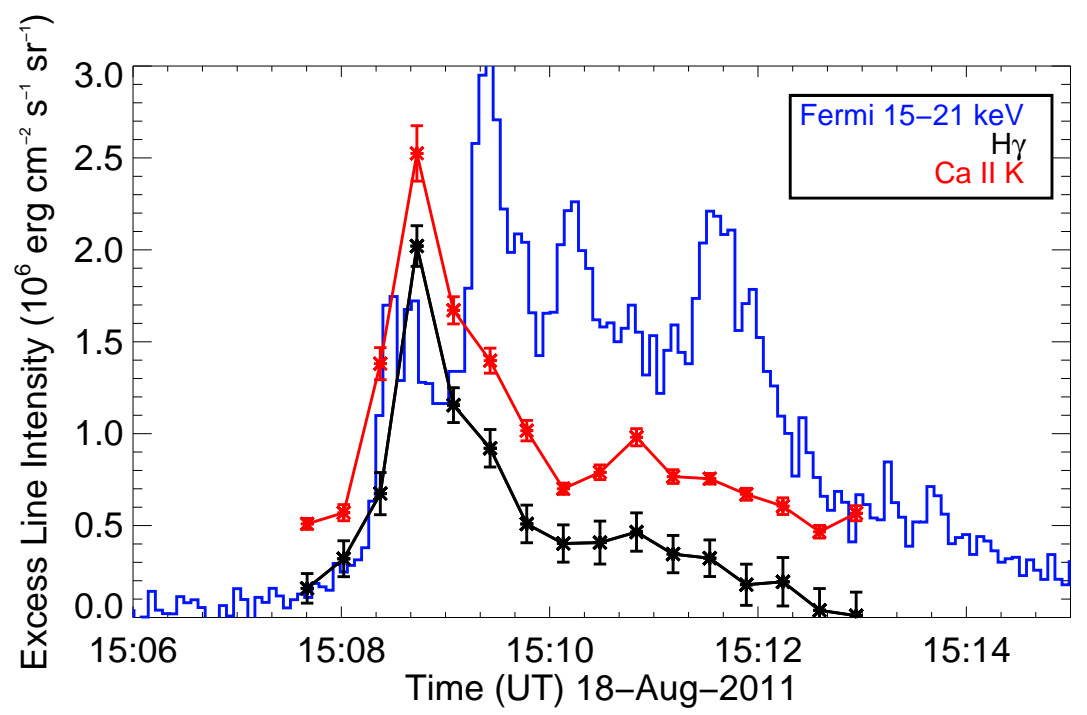

Fig. 11. - The light curves of $\mathrm{Ca}$ II $\mathrm{K}$, and $\mathrm{H} \gamma$ for a region in the plage flare ribbon in the 18th slit position of the raster compared to the Fermi $15-21 \mathrm{keV}$ light curve. The emission line values were obtained from the ribbon intersected by the slit corresponding to the rightmost vertical white line in Figure 1 (bottom left panel). The average excess line intensity is calculated over the three brightest spatial pixels in order to facilitate comparison to the umbral kernel (Figure 10). 


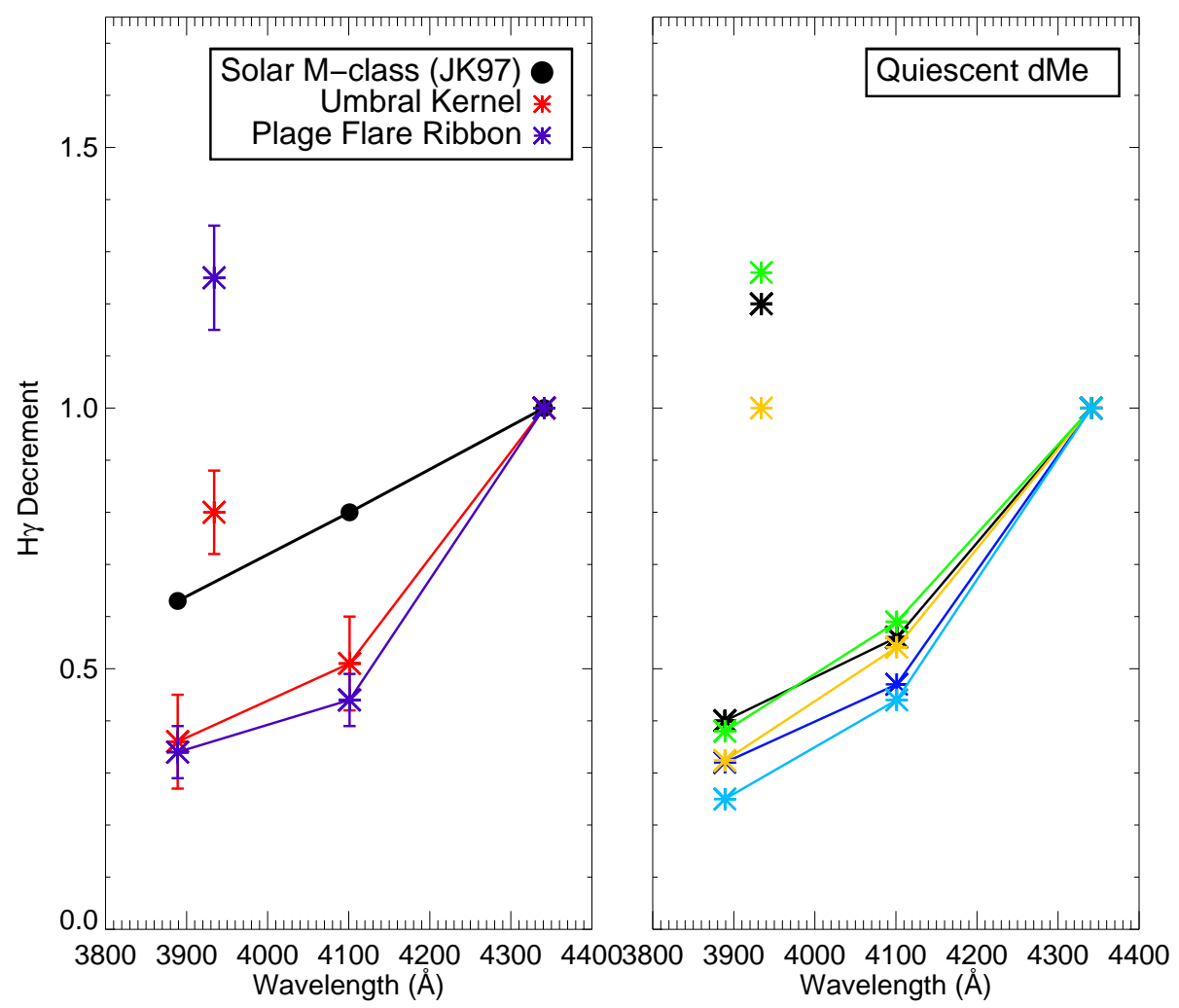

Fig. 12.- Left: The Balmer decrements for the plage flare ribbon at maximum line emission 15:08:44 (purple) and umbral kernel at 15:09:30 (red) compared to the decrements from the M7.7 solar flare presented in Johns-Krull et al. (1997). The decrements are shown as the wavelength-integrated emission in $\mathrm{H} 8, \mathrm{Ca}$ II $\mathrm{K}, \mathrm{H} \delta$ divided by that in $\mathrm{H} \gamma$; the lines connect only the hydrogen Balmer series. The decrements were obtained from the emission line averaged over the same three pixels as used for continuum detection and analysis. Right: The Balmer decrements of quiescent dMe spectra obtained from the literature (black: AD Leo from Hawley \& Pettersen (1991); orange: AD Leo from Kowalski et al. (2013); green: UV Ceti from Phillips et al. (1988); light blue: YZ CMi from Doyle et al. (1988); dark blue: AT Mic from García-Alvarez et al. (2002)). 


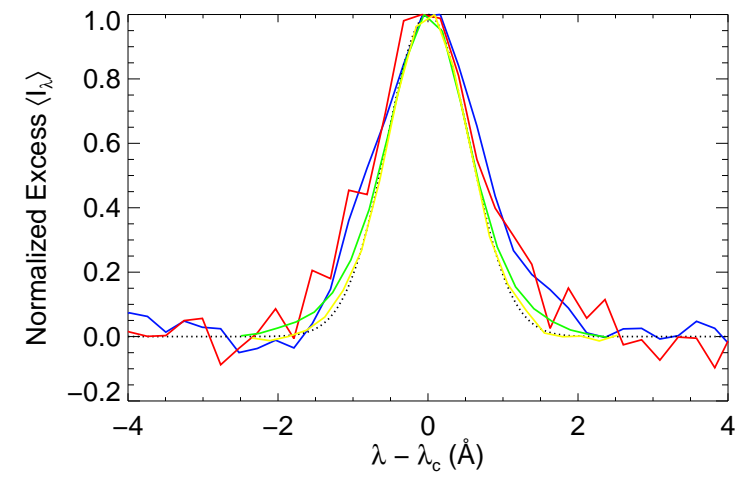

Fig. 13. - The H8 line profile of the excess emission in umbral kernel (at 15:09:30; red) and plage flare ribbon (at 15:08:44; blue), normalized to their peak excess intensity. A gaussian with the instrumental FWHM of $1.3 \AA$ is shown as a dotted line, and the Ca II K line profiles are shown at the same times and locations (plage flare ribbon as green, umbral kernel as yellow) as for the $\mathrm{H} 8$ profiles. 


\section{Appendix A: Intensity Calibration}

In this Appendix, we describe the detailed spectral reduction procedure and intensity calibration. De-focused quiescent solar spectra were obtained away from the active region at UT 16:26 (airmass of 1.34) at the same heliocentric radius vector (0.68) and DST guider angle (119.8 deg) as the observations. To isolate the CCD variations from the quiescent solar spectrum, we performed a median filtering which resulted in a master flat field image. This master flat was divided out of all images.

Wavelength and intensity calibration was carried out using the disk-center absolute solar intensity spectrum obtained with the Fourier Transform Spectrometer (FTS) with spectral resolution $\mathrm{R}=350000$ (Neckel 1999). The wavelength solution and spectral resolution were obtained by aligning the quiescent solar spectral features in the HSG spectra to the FTS spectral features. The HSG dispersions are $0.28 \AA$ pixel $^{-1}, 0.24 \AA$ pixel $^{-1}$,

$0.35 \AA$ pixel $^{-1}$, and $0.35 \AA$ pixel $^{-1}$ for the bluest to the reddest cameras, respectively. We found that the spectral resolution was approximately $0.9-1.2 \AA$ at $4300 \AA$ ( R 4000) by convolving the FTS spectrum with Gaussians of various widths.

To calibrate the active region spectra to an absolute intensity scale, we used the quiescent solar spectrum from UT 16:26 (with flat-field variations removed) as the reference. From this, we extracted an average solar spectrum over 10 spatial pixels, which was converted from counts spatial pixel ${ }^{-1}$ wavelength pixel ${ }^{-1}$ to counts $\mathrm{sr}^{-1}$ wavelength pixel ${ }^{-1}$ by multiplying by $4.25 \times 10^{10} \operatorname{arcseconds}^{2} \mathrm{sr}^{-1} \times 1$ spatial pixel $/ 0^{\prime \prime} .39 \times 1 / 0^{\prime \prime} .67$ (pixel size along the slit, and slit size, respectively).

The IRAF routines standard and sensfunc were used to determine the instrumental sensitivity. These routines divide the reference solar spectrum (in units of counts $\mathrm{sr}^{-1}$ wavelength pixel $^{-1}$ ) by the exposure time and user-defined wavelength bins in order to compare against the FTS disk-center intensity spectrum averaged over the same wavelength 
bins. The FTS spectrum was multiplied by the limb darkening $(D)$ corresponding to a radius vector of 0.68 , and was converted to $\mathrm{AB}$ magnitudes $\left(m_{\mathrm{AB}}=-2.5 \log _{10}\left(I_{\nu} D\right)-48.60\right)$ for the IRAF routines. The limb darkening was determined to be 0.83 from equation 8 of Pierce \& Slaughter (1977) using $\mu=0.74^{5}$. The limb darkening is wavelength dependent, but over our spectral range varies only by about $1 \%$, so we used a constant limb darkening. We used an atmospheric extinction curve obtained from a nearby site at the Apache Point Observatory. The instrumental sensitivity was fit with a smooth function in $10 \AA$ wide bins where the FTS spectrum was free of strong absorption lines.

We used the resultant instrumental sensitivity function to calculate the wavelengthdependent conversion factor, $X(\lambda)$, which converts the images in units of counts s $\mathrm{sr}^{-1} \AA^{-1}$ to intensity in units of ergs s $\mathrm{sm}^{-1} \mathrm{sr}^{-1} \AA^{-1}$ at the airmass (1.34) of the quiescent reference spectral observation. The conversion factor, $X(\lambda)$, which has units of $\operatorname{ergs~} \mathrm{cm}^{-2} \mathrm{count}^{-1}$, was adjusted for the wavelength-dependent atmospheric transmission at the airmass of the target (active region) observation. This was done by multiplying $X(\lambda)$ by $T(\lambda)_{\text {ref }} / T(\lambda)_{\operatorname{targ}}$, where $T(\lambda)$ is the atmospheric transmission at a given airmass (i.e., $T(\lambda)=10^{E(\lambda) \sec z /-2.5}$ where $E(\lambda)$ is the atmospheric extinction in units of magnitudes airmass ${ }^{-1}$ and sec $\mathrm{z}$ is the airmass of the observation). Before applying this final conversion factor, the spectra were aligned to a common spatial orientation and interpolated to a common pixel scale, $0^{\prime \prime} .39$ pixel $^{-1}$. Within each camera's spectral range, wavelength-dependent shifts of $0.5-2$ pixels were also applied to account for differential refraction. This calibration procedure was performed for all slit positions of all CCD's, resulting in a $2 \mathrm{D}$ image with wavelength and spatial pixel axes, having units of $\left[I_{\lambda, \mu=0.74}\right]$.

\footnotetext{
${ }^{5}$ We used Sykes (1953) second-order fit to $\ln \mu$ given in Pierce \& Slaughter (1977); the fifth order fit to $\ln \mu$ in Pierce \& Slaughter (1977) gives a slightly larger amount of limb darkening, 0.80 .
} 
Table 1. Horizontal Spectrograph Instrumental Setup

\begin{tabular}{lccccc}
\hline \hline CCD & Wavelength Range $[\AA]$ & Useable Wavelength Range† $[\AA]$ & Dispersion $\left[\AA\right.$ pix $\left.{ }^{-1}\right]$ & Exp Time $[\mathrm{ms}]$ & Pixel Scale $\left[{ }^{\prime \prime}\right.$ pix $\left.{ }^{-1}\right]$ \\
\hline cc06 & $3500-3790$ & $3654-3674$ & 0.28 & 500 & 0.39 \\
cc01 & $3771-4020$ & $3830-3978$ & 0.24 & 40 & 0.34 \\
ccc07 & $3945-4306$ & $4085-4125$ & 0.35 & 20 & 0.48 \\
ccc08 & $4198-4559$ & $4213-4553$ & 0.35 & 10 & 0.48 \\
\hline
\end{tabular}

Note. - †These correspond to wavelength ranges that are useable for spectral characterization (e.g., slope determination). For ccc06 and ccc07 we display in the figures larger spectral ranges of 3600-3740 $\AA$ and 4016-4200 $\AA$ for only the purposes of white-light detection (see comments on chromatic aberration in Section 2.4. 


\section{REFERENCES}

Abbett, W. P., \& Hawley, S. L. 1999, ApJ, 521, 906

Acampa, E., Smaldone, L. A., Sambuco, A. M., \& Falciani, R. 1982, A\&AS, 47, 485

Airapetian, V. S., \& Holman, G. D. 1998, ApJ, 501, 805

Allred, J. C., Hawley, S. L., Abbett, W. P., \& Carlsson, M. 2005, ApJ, 630, 573

Boyer, R., Sotirovsky, P., Machado, M. E., \& Rust, D. M. 1985, Sol. Phys., 98, 255

Canfield, R. C., Penn, M. J., Wulser, J.-P., \& Kiplinger, A. L. 1990, ApJ, 363, 318

Carlsson, M., \& Stein, R. F. 1994, in Chromospheric Dynamics, ed. M. Carlsson, 47

Carlsson, M., \& Stein, R. F. 1995, ApJ, 440, L29

Carlsson, M., \& Stein, R. F. 1997, ApJ, 481, 500

Cauzzi, G., Falchi, A., Falciani, R., \& Smaldone, L. A. 1996, A\&A, 306, 625

Cauzzi, G., Falchi, A., Falciani, R., Smaldone, L. A., Schwartz, R. A., \& Hagyard, M. 1995, A\&A, 299, 611

Cavallini, F. 2006, Sol. Phys., 236, 415

Chalabaev, A., \& Maillard, J. P. 1983, A\&A, 127, 279

Cheng, J. X., Ding, M. D., \& Carlsson, M. 2010, ApJ, 711, 185

Deng, N., et al. 2013, ApJ, 769, 112

Donati-Falchi, A., Falciani, R., \& Smaldone, L. A. 1985, A\&A, 152, 165

Donati-Falchi, A., Smaldone, L. A., \& Falciani, R. 1984, A\&A, 131, 256 
Doyle, J. G., Butler, C. J., Bryne, P. B., \& van den Oord, G. H. J. 1988, A\&A, 193, 229

Drake, S. A., \& Ulrich, R. K. 1980, ApJS, 42, 351

Emslie, A. G., et al. 2012, ApJ, 759, 71

Emslie, A. G., Miller, J. A., \& Brown, J. C. 2004, ApJ, 602, L69

Falchi, A., \& Mauas, P. J. D. 2002, A\&A, 387, 678

Falchi, A., Qiu, J., \& Cauzzi, G. 1997, A\&A, 328, 371

Fisher, G. H. 1989, ApJ, 346, 1019

Fletcher, L., Hannah, I. G., Hudson, H. S., \& Metcalf, T. R. 2007, ApJ, 656, 1187

Fletcher, L., \& Hudson, H. S. 2008, ApJ, 675, 1645

Fletcher, L., Kowalski, A., Cauzzi, G., Hawley, S. L., \& Hudson, H. S. 2013, in AAS/Solar Physics Division Meeting, Vol. 44, AAS/Solar Physics Division Meeting, 100.67

Fuhrmeister, B., Liefke, C., Schmitt, J. H. M. M., \& Reiners, A. 2008, A\&A, 487, 293

García-Alvarez, D., Jevremović, D., Doyle, J. G., \& Butler, C. J. 2002, A\&A, 383, 548

Güdel, M. 1997, ApJ, 480, L121

Güdel, M., Audard, M., Kashyap, V. L., Drake, J. J., \& Guinan, E. F. 2003, ApJ, 582, 423

Hawley, S. L., \& Fisher, G. H. 1992, ApJS, 78, 565

Hawley, S. L., \& Fisher, G. H. 1994, ApJ, 426, 387

Hawley, S. L., \& Pettersen, B. R. 1991, ApJ, 378, 725

Heinzel, P., \& Kleint, L. 2014, ApJ, 794, L23 
Hiei, E. 1982, Sol. Phys., 80, 113

Hudson, H. S. 1972, Sol. Phys., 24, 414

Hudson, H. S., Acton, L. W., Hirayama, T., \& Uchida, Y. 1992, PASJ, 44, L77

Hudson, H. S., Wolfson, C. J., \& Metcalf, T. R. 2006, Sol. Phys., 234, 79

Ichimoto, K., \& Kurokawa, H. 1984, Sol. Phys., 93, 105

Jess, D. B., Mathioudakis, M., Christian, D. J., Keenan, F. P., Ryans, R. S. I., \& Crockett, P. J. 2010, Sol. Phys., 261, 363

Jess, D. B., Mathioudakis, M., Crockett, P. J., \& Keenan, F. P. 2008, ApJ, 688, L119

Johns-Krull, C. M., Hawley, S. L., Basri, G., \& Valenti, J. A. 1997, ApJS, 112, 221

Kane, S. R., Love, J. J., Neidig, D. F., \& Cliver, E. W. 1985, ApJ, 290, L45

Kashyap, V. L., Drake, J. J., Güdel, M., \& Audard, M. 2002, ApJ, 580, 1118

Kerr, G. S., \& Fletcher, L. 2014, ApJ, 783, 98

Kowalski, A. F., Hawley, S. L., Wisniewski, J. P., Osten, R. A., Hilton, E. J., Holtzman, J. A., Schmidt, S. J., \& Davenport, J. R. A. 2013, ApJS, 207, 15

Kretzschmar, M. 2011, A\&A, 530, A84

Krucker, S., Hudson, H. S., Jeffrey, N. L. S., Battaglia, M., Kontar, E. P., Benz, A. O., Csillaghy, A., \& Lin, R. P. 2011, ApJ, 739, 96

Kunkel, W. E. 1970, ApJ, 161, 503

Kurokawa, H., Takakura, T., \& Ohki, K. 1988, PASJ, 40, 357

Leenaarts, J., Rutten, R. J., Carlsson, M., \& Uitenbroek, H. 2006, A\&A, 452, L15 
Lemen, J. R., et al. 2012, Sol. Phys., 275, 17

Linsky, J. L., \& Wood, B. E. 1994, ApJ, 430, 342

Livshits, M. A., Badalian, O. G., Kosovichev, A. G., \& Katsova, M. M. 1981, Sol. Phys., 73,269

Machado, M. E., Emslie, A. G., \& Avrett, E. H. 1989, Sol. Phys., 124, 303

Machado, M. E., Emslie, A. G., \& Brown, J. C. 1978, Sol. Phys., 58, 363

Machado, M. E., \& Rust, D. M. 1974, Sol. Phys., 38, 499

Meegan, C., et al. 2009, ApJ, 702, 791

Metcalf, T. R., Alexander, D., Hudson, H. S., \& Longcope, D. W. 2003, ApJ, 595, 483

Neckel, H. 1999, Sol. Phys., 184, 421

Neidig, D. F. 1983, Sol. Phys., 85, 285

Neidig, D. F. 1989, Sol. Phys., 121, 261

Neidig, D. F., Grosser, H., \& Hrovat, M. 1994, Sol. Phys., 155, 199

Neidig, D. F., \& Kane, S. R. 1993, Sol. Phys., 143, 201

Neidig, D. F., Kiplinger, A. L., Cohl, H. S., \& Wiborg, P. H. 1993, ApJ, 406, 306

Neidig, D. F., \& Wiborg, P. H., Jr. 1984, Sol. Phys., 92, 217

Osten, R. A., Hawley, S. L., Allred, J., Johns-Krull, C. M., Brown, A., \& Harper, G. M. 2006, ApJ, 647, 1349

Petrosian, V., \& Liu, S. 2004, ApJ, 610, 550 
Phillips, K. J. H., Bromage, G. E., Dufton, P. L., Keenan, F. P., \& Kingston, A. E. 1988, MNRAS, 235, 573

Pierce, A. K., \& Slaughter, C. D. 1977, Sol. Phys., 51, 25

Radziszewski, K., Rudawy, P., \& Phillips, K. J. H. 2011, A\&A, 535, A123

Reardon, K. P. 2006, Sol. Phys., 239, 503

Rimmele, T. R. 2004, in Society of Photo-Optical Instrumentation Engineers (SPIE) Conference Series, Vol. 5490, Advancements in Adaptive Optics, ed. D. Bonaccini Calia, B. L. Ellerbroek, \& R. Ragazzoni, 34

Russell, A. J. B., \& Fletcher, L. 2013, ApJ, 765, 81

Sharykin, I. N., \& Kosovichev, A. G. 2014, ApJ, 788, L18

Sykes, J. B. 1953, MNRAS, 113, 198

Švestka, Z. 1963, Bulletin of the Astronomical Institutes of Czechoslovakia, 14, 234

Švestka, Z. 1970, Sol. Phys., 13, 471

Watanabe, K., Shimizu, T., Masuda, S., Ichimoto, K., \& Ohno, M. 2013, ApJ, 776, 123

Worden, S. P., Schneeberger, T. J., Giampapa, M. S., Deluca, E. E., \& Cram, L. E. 1984, ApJ, 276, 270

Zirin, H. 1980, ApJ, 235, 618

Zirin, H., \& Neidig, D. F. 1981, ApJ, 248, L45

This manuscript was prepared with the AAS IATEX macros v5.2. 